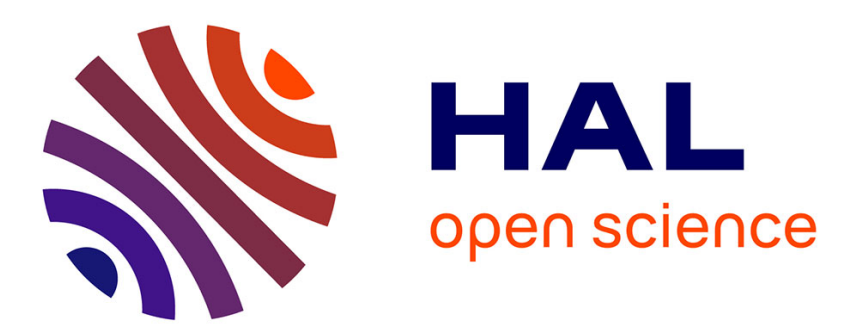

\title{
Signature and energetics of internal gravity waves in stratified turbulence
}

\author{
Andrea Maffioli, Alexandre Delache, Fabien S. Godeferd
}

\section{To cite this version:}

Andrea Maffioli, Alexandre Delache, Fabien S. Godeferd. Signature and energetics of internal gravity waves in stratified turbulence. Physical Review Fluids, 2020, 5 (11), pp.114802. 10.1103/PhysRevFluids.5.114802 . hal-03047112

\section{HAL Id: hal-03047112 \\ https://hal.science/hal-03047112}

Submitted on 3 Jun 2021

HAL is a multi-disciplinary open access archive for the deposit and dissemination of scientific research documents, whether they are published or not. The documents may come from teaching and research institutions in France or abroad, or from public or private research centers.
L'archive ouverte pluridisciplinaire HAL, est destinée au dépôt et à la diffusion de documents scientifiques de niveau recherche, publiés ou non, émanant des établissements d'enseignement et de recherche français ou étrangers, des laboratoires publics ou privés. 


\title{
Signature and energetics of internal gravity waves in stratified turbulence
}

\author{
Andrea Maffioli $\odot,{ }^{1, *}$ Alexandre Delache, ${ }^{2}$ and Fabien S. Godeferd ${ }^{1}$ \\ ${ }^{1}$ Laboratoire de Mécanique des Fluides et d'Acoustique, Université de Lyon, Ecole Centrale de Lyon, \\ INSA de Lyon, Université Claude Bernard Lyon 1, CNRS, France \\ ${ }^{2}$ Laboratoire de Mécanique des Fluides et d'Acoustique, site de Saint-Etienne, Université de Lyon, \\ Université Jean Monnet de Saint-Etienne, CNRS, France
}

(Received 16 December 2019; accepted 30 September 2020; published 11 November 2020)

\begin{abstract}
Internal gravity waves propagating within homogeneous stratified turbulence are the subject of the present study. A spatiotemporal analysis is carried out on the results of direct numerical simulations including a forcing term, with the aim of showing the energy content of the simulations as a function of frequency, $\omega$, and wave-vector inclination to the horizontal, $\theta$. Clear signatures of the dispersion relation of internal gravity waves, $\omega=$ $\pm N \cos \theta$, where $N$ is the Brunt-Väisälä frequency, are observed in all our simulations, which have low Froude number, $\mathrm{Fr}_{h} \ll 1$, and increasing buoyancy Reynolds number up to $\operatorname{Re}_{b} \approx 10$. Interestingly, we observe the presence of high-frequency waves with $\omega \sim N$ and a corresponding low-frequency vortex mode, both containing a non-negligible amount of energy. These waves are large-scale waves, their energy signature being found at scales larger than the forcing scales. We also observe the growth of energy in the shear modes, constituting a horizontal mean flow, and we show that their continuous growth is due to an upscale energy transfer, from the forcing scales to larger horizontal as well as vertical scales. These shear modes are found to be responsible for Doppler shifting the frequency of the large-scale waves. When considering the wave energy across the simulations at varying $\mathrm{Re}_{b}$, such energy is seen to reduce as $\mathrm{Re}_{b}$ is increased and the flow enters the strongly stratified turbulence regime. The classical wave-vortex decomposition, based on a purely spatial decomposition of instantaneous snapshots of the flow, is analyzed within the current framework and is seen to correspond relatively well to the "true" wave signal identified by the spatiotemporal analysis, at least for the large-scale waves with $\omega \sim N$. Distinct energy peaks in $\theta-\omega$ space highlight that the waves have preferential directions of propagation, specifically $\theta=45^{\circ}$ and $\theta \approx 55^{\circ}$, similar to observations in studies of wave radiation from localized regions of turbulence. This suggests that the same wave-generation mechanisms may be relevant for homogeneous and inhomogeneous stratified turbulent flows.
\end{abstract}

DOI: 10.1103/PhysRevFluids.5.114802

\section{INTRODUCTION}

Stratified flows are ubiquitous in geophysical fluid dynamics and the stable stratification in such flows often has a leading-order influence on the dynamics. As is well known, the stable vertical density variation in stratified flows leads to the existence of internal gravity waves. These waves are dispersive and anisotropic since their frequency, phase velocity, and group velocity strongly depend on their direction of propagation. The frequency of internal gravity waves is given by the dispersion relation, $\omega= \pm N k_{h} / k$, where $k=|\mathbf{k}|$ is the magnitude of the wave vector

*andrea.maffioli@ec-lyon.fr 
$\mathbf{k}=\left[k_{x}, k_{y}, k_{z}\right]$ and $k_{h}=\sqrt{k_{x}^{2}+k_{y}^{2}}$ is the horizontal wave number. The Brunt-Väisälä frequency is $N=\sqrt{-\left(g / \rho_{0}\right) d \bar{\rho} / d z}$, where $d \bar{\rho} / d z$ is the background density gradient, $g$ is gravity, and $\rho_{0}$ is a reference density. Advective motions, such as turbulent eddies, of course are also present in stratified flows. In stratified turbulence, these two types of motion coexist in the same region of space, and they evolve and interact chaotically. Because of this highly complex picture, it is a task of chief difficulty to separate the dynamics of one motion from the other.

A number of studies [1-4] have used a classical decomposition called wave-vortex decomposition to disentangle waves from vortices. The wave-vortex decomposition was proposed early on in the study of stratified turbulence [5,6]; it consists of a spatial decomposition of an instantaneous field of stratified turbulence, given by the velocity field $\mathbf{u}(\mathbf{x})$, into a wave component $\mathbf{u}_{w}(\mathbf{x})$ and a vortex component $\mathbf{u}_{v}(\mathbf{x})$. The analysis relies on the two types of motion evolving on different timescales: The waves evolve on the "fast" timescale $N^{-1}$ and the vortices evolve on the "slow" timescale $\tau$, the eddy turnover time [7]. Here the eddy turnover time can be taken as $\tau=\ell_{h} / u_{h}$, where $\ell_{h}$ and $u_{h}$ are typical horizontal length and velocity scales of the turbulent flow. An analogous decomposition has been put forward for flows which are both stratified and rotating [8], and this decomposition has been used to distinguish waves from turbulence in such flows [8-10]. The main limitation of applying the wave-vortex decomposition to stratified turbulence is that, if the Reynolds number is high, the consequent high nonlinearity of the flow may prevent a clear timescale separation between waves and vortices. In particular, this could be the case for the strongly stratified turbulence (SST) regime $[11,12]$, a dynamical regime which is encountered in the concurrent limits of low horizontal Froude number, $\operatorname{Fr}_{h}=u_{h} / N \ell_{h} \ll 1$, and high buoyancy Reynolds number, $\operatorname{Re}_{b}=\epsilon / \nu N^{2} \gg 1$ [13] (here $\epsilon$ is an average kinetic energy dissipation rate). This regime is particularly relevant as its predictions are consistent with turbulence observed in the ocean and in the atmosphere, far away from solid boundaries [14-16]. As identified in a scaling analysis by Ref. [11], the turbulent structures here consist of layers with a low aspect ratio, $\ell_{v} / \ell_{h} \sim u_{h} / N \ell_{h}=F r_{h}$, where $\ell_{v}$ is the vertical length scale, which is set by the stratification to be the buoyancy length scale $u_{h} / N$. Within this framework, it is straightforward to show that waves of the above aspect ratio, $\ell_{v} / \ell_{h}$, will have wave number $k \sim k_{v}$ and frequency $\omega \sim N k_{h} / k_{v} \sim N \ell_{v} / \ell_{h} \sim \tau^{-1}$ [11]. Therefore, waves and vortices both have a timescale $\tau$ and so they probably strongly interact. The two-timescale assumption underlying the wave-vortex decomposition would thus break down in the SST regime, making the application of the decomposition and its physical meaning questionable. This limitation led Lindborg and Brethouwer [14] to take an alternative route toward assessing the wave content of a stratified turbulent flow. In their work, they computed frequency spectra of individual Fourier modes $\hat{\mathbf{u}}(\mathbf{k})$ at selected wave vectors $\mathbf{k}$ in numerical simulations of stratified turbulence in the SST regime. This allowed them to focus on the dispersion relation frequency $|\omega|=N k_{h} / k$ and check whether it corresponded to strong energy peaks in the frequency spectra, demonstrating the presence of internal gravity waves. The study highlighted clear signatures of internal gravity waves if $\mathbf{k}$ corresponded to large scales of the turbulent flow and to aspect ratios $\ell_{v} / \ell_{h}$ being not much smaller than unity; at smaller scales and/or at very small $\ell_{v} / \ell_{h}$ this wave signature was less clear or absent. Another finding of this study was that at large scales the wave-vortex decomposition worked well [14]. It is worth noting that waves with $\ell_{v} / \ell_{h} \sim k_{h} / k_{v} \sim 1$ have a frequency $\omega \sim N$, i.e., a relatively high frequency, unlike what was suggested for SST [11].

In recent years, a more detailed analysis has been applied to study waves in fluid mechanics, made feasible by the advances in computational power. It consists of a spatiotemporal analysis of a wave-bearing flow field, in which time series of snapshots of the flow are transformed into wavenumber-frequency space via a Fourier transform. For example, if the flow field is three-dimensional (3D) with coordinates $x, y, z$, a quantity $f=f(x, y, z, t)$ is transformed via a four-dimensional (4D) Fourier transform into the quantity $\tilde{f}=\tilde{f}\left(k_{x}, k_{y}, k_{z}, \omega\right)$. This allows one to focus on the portion of the 4D space described by the wave dispersion relation, $\omega=\omega\left(k_{x}, k_{y}, k_{z}\right)$, to look for signatures of the wave field in the problem and, potentially, to quantify the energy $|\tilde{f}|^{2}$ contained in the waves. This procedure is conceptually the same as that of Lindborg and Brethouwer [14], who applied 
it to individual spatial Fourier modes; such an approach is now extended to the entirety of the Fourier modes in an experiment or simulation. Because of the computational cost of calculating $3 \mathrm{D}$ or 4D Fourier transforms of high-resolution data, the full analysis has been carried out only in a number of studies of turbulent flows, for example, in two recent experimental investigations: One on gravity-capillary waves on the surface of a fluid [17] and another on inertial waves in 3D rotating turbulence [18]. Both these studies found clear signatures of the wave dispersion relation in their experimental data, with an accumulation of energy on and around the dispersion relation. The full spatiotemporal analysis has also been carried out using data from numerical simulations, in direct numerical simulation (DNS) studies of internal gravity waves in stratified turbulence [19] and inertial waves in rotating turbulence [20,21], and in a recent study of inertial waves in 3D geodynamo simulations [22]. In a number of other studies, reduced analyses have been performed, focusing on frequency spectra of extended regions of Fourier space, e.g., studies of internal gravity waves in stratified turbulence [23], inertial waves in rotating turbulence [24], and Alfvén waves in magnetohydrodynamic turbulence [25]. Focusing on the case of interest, in a recent study [23], results from DNS of stratified turbulence forced using a periodic tidal deformation were presented, in which the Fourier modes were reduced according to their polar angle $\phi=\tan ^{-1}\left(k_{h} / k_{z}\right)$ in order to obtain (after a time Fourier transform) the kinetic energy as a function of frequency and $\phi$, $E_{K}(\phi, \omega)$. A clear signature of the dispersion relation, $\omega= \pm N \sin \phi$, was observed in $E_{K}(\phi, \omega)$ over all internal gravity wave frequencies, $0 \leqslant|\omega| \leqslant N$. This study was, however, limited to low values of the buoyancy Reynolds number, $\operatorname{Re}_{b}<1$, so the DNS were probably in a viscously dominated state and not in the SST regime [13]. Another recent numerical study of internal gravity waves in stratified flows [19] used a full 4D spatiotemporal analysis of a DNS run at $\operatorname{Re}_{b}=1$; they obtained wave signatures from their analysis which was also employed to reconstruct wave and eddy fields in physical space by filtering out relevant portions of the k- $\omega$ space. Overall, the work to date on this subject leaves room for further study of stratified turbulence using a spatiotemporal analysis, this time at $\operatorname{Re}_{b} \gtrsim 1$, to consider the internal gravity wave content of the turbulence in a regime close to the SST regime. It is worth underlining that an advantage of a spatiotemporal analysis compared to a purely spatial analysis, such as the wave-vortex decomposition, is that significantly more information is ultimately available, in particular with respect to the energy content as a function of frequency. Also, it is important to note that while detailed spatiotemporal analyses are a recent tool in fundamental fluid mechanics studies, they are a relatively well-established tool in the analysis of large-scale atmospheric data, e.g., for the extraction of signatures of equatorial waves using large-scale and long-term satellite observations [26].

The key significance of studying waves in SST is that it is not clear what type of waves, if any, are excited by the layers and how important they are in terms of their energy content. The only prediction regarding waves in SST is that we may expect waves with low aspect ratio $\ell_{v} / \ell_{h}$, where $\ell_{v}$ and $\ell_{h}$ are typical vertical and horizontal scales (or wavelengths), similar to the turbulent layers [11]. As mentioned previously, such waves correspond to low-frequency wave motions with $\omega \sim \tau^{-1}$, which opens up the possibility of strong nonlinear interactions between these low-frequency waves and the turbulent layers (evolving on the same timescale). A number of open questions remain:

(1) Are we able to observe such low-frequency waves in DNS using spatiotemporal analysis?

(2) Are waves of other frequencies within $0 \leqslant \omega \leqslant N$ also present, confirming the observations of Lindborg and Brethouwer [14]?

(3) How important are the waves in the global energetics of stratified turbulence?

(4) How are they generated within a highly turbulent flow such as SST?

(5) Does the wave-vortex decomposition give us insight into the wave content of SST?

(6) Do the characteristics of the waves depend on the way the turbulence is forced or on the subdivision of the force between wave and vortex components of the flow?

While there are relatively few studies of waves in homogeneous stratified turbulence, the problem of internal gravity wave radiation from a localized turbulent region has been treated more extensively. Previous studies include experiments and simulations of a stratified or unstratified turbulent region exciting waves in adjacent quiescent stratified regions [27,28] and experimental [29] and 
numerical $[30,31]$ studies of turbulent wakes behind a sphere towed in a stratified fluid. All these studies observed the presence of turbulence-generated waves in the surrounding fluid. Moreover, the internal gravity waves were consistently found to have preferential directions of propagation. This is the case, for example, of the experimental study of a stratified turbulent wake [29], where waves were observed with a single propagation direction, $\theta=90^{\circ}-\phi \approx 55^{\circ}$. This particular propagation direction was observed also in other studies but often within a range of propagation angles, such as in the experimental study of turbulence generated above a quiescent stratified fluid [27], in which $\theta=42-55^{\circ}$ is reported. Justifications have been put forward for preferential orientations of internal gravity waves in this problem. For example, Dohan and Sutherland [27] propose that a propagation at $\theta=45^{\circ}$ maximizes the vertical transport of horizontal momentum while $\theta=35^{\circ}$ maximizes the vertical transport of energy, so that a resonant feedback between waves and turbulence would ultimately lead to the selection of these angles. The interesting question is then whether some of these results carry over to internal gravity waves in the interior of stratified turbulence and whether we can draw parallels between the wave radiation in these two different classes of stratified turbulent flows.

In what follows, we will present results from a spatiotemporal analysis of DNS of stratified turbulence at $\operatorname{Re}_{b} \gtrsim 1$, revealing the internal gravity waves in the simulations. The aim is to try and give answers to some of the open questions in this important problem.

\section{METHODS}

\section{A. Spatiotemporal analysis}

\section{Reduced analysis}

In this paper, we will explore a detailed analysis tool of wave-bearing flows in which both spatial and temporal information on the flow field are used, which distinguishes this tool from an analytical but purely spatial tool such as the wave-vortex decomposition. As mentioned in Sec. I, a full spatiotemporal analysis of a 3D flow field requires one to accumulate time series of the 3D fields and then to perform a 4D Fourier transform, taking fields that depend on space and time into wave-number-frequency space, $f(x, y, z, t) \Rightarrow \tilde{f}\left(k_{x}, k_{y}, k_{z}, \omega\right)$. Typically, such fields are obtained from DNS that are based on a pseudospectral method, in which spatial Fourier transforms are used. In this case, the fields are already in wave-number space, as the DNS code solves for $\hat{f}\left(k_{x}, k_{y}, k_{z}, t\right)$. Even in this situation, however, taking the final Fourier transform of the 4D fields from temporal to frequency space is a computationally intensive task that can become prohibitive as the spatial (and temporal) resolution of the DNS increases.

In the present work, we follow Ref. [23] and perform a reduced spatiotemporal analysis, which is less expensive computationally. As briefly mentioned before, the first step consists in summing the velocity Fourier modes, $\hat{\mathbf{u}}\left(k_{x}, k_{y}, k_{z}\right)$, at a given time instant, over "conical shells" at a constant angle with respect to the vertical axis. Contrary to Ref. [23], we work with the equatorial angle $\theta$ and not with the polar angle; the equatorial angle is defined as $\theta=\tan ^{-1}\left(k_{z} / k_{h}\right)$. In terms of $\theta$, the dispersion relation (DR) of internal gravity waves is given by $\omega=\omega_{D R}$, where $\omega_{D R}= \pm N \cos \theta$. The summation over conical shells leads to the formation of the quantities $\mathcal{U}(\theta)$ and $\mathcal{A}(\theta)$ :

$$
\mathcal{U}(\theta)=\sum_{\theta-\Delta \theta / 2}^{\theta+\Delta \theta / 2} \hat{\mathbf{u}}\left(k_{x}, k_{y}, k_{z}\right), \quad \mathcal{A}(\theta)=\sum_{\theta-\Delta \theta / 2}^{\theta+\Delta \theta / 2}\left(\frac{g}{\rho_{0}}\right) \hat{\rho}^{\prime}\left(k_{x}, k_{y}, k_{z}\right),
$$

where $\Delta \theta$ is the increment in $\theta$, which we will take either as $0.5^{\circ}$ or $1^{\circ}$. In the second equation, $\hat{\rho}^{\prime}$ is the Fourier transform of $\rho^{\prime}$, the density perturbation away from the linear stratification, which itself is given by $\rho_{0}+\bar{\rho}(z)$. Note that $\mathcal{U}(\theta)$ is a vector quantity while $\mathcal{A}(\theta)$ is a scalar quantity and both are complex valued. For an illustration of this procedure, see Fig. 1. Subsequently, time series of $\mathcal{U}(\theta)$ and $\mathcal{A}(\theta)$ can be accumulated and they are then transformed into frequency space, e.g., $\mathcal{U}(\theta, t) \Rightarrow \mathcal{U}(\theta, \omega)$. Finally, it is convenient to form the kinetic and potential energy in 


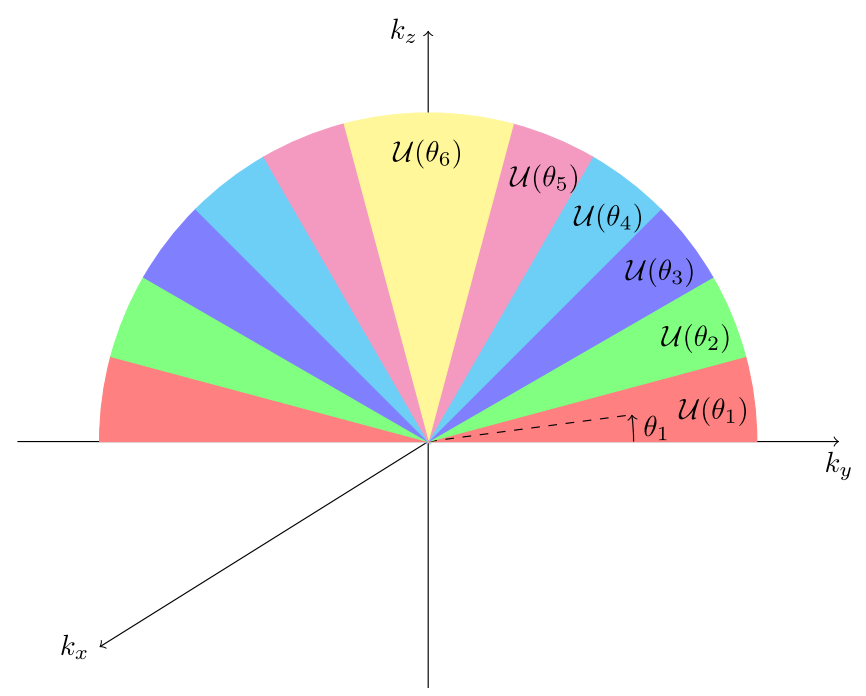

FIG. 1. Illustration of conical shells and their corresponding Fourier mode sums, $\mathcal{U}(\theta)$, shown on a cut at $k_{x}=0$. Note that modes on the $k_{z}$ axis corresponding to the shear modes are put into a separate individual shell. The same applies to modes on the horizontal $k_{x}-k_{y}$ plane.

angle-frequency space from $\mathcal{U}(\theta, \omega)$ and $\mathcal{A}(\theta, \omega)$ :

$$
E_{K}(\theta, \omega)=\frac{1}{2} \mathcal{U}(\theta, \omega) \cdot \mathcal{U}(\theta, \omega)^{*}, \quad E_{P}(\theta, \omega)=\frac{1}{2 N^{2}}|\mathcal{A}(\theta, \omega)|^{2} .
$$

These quantities are well suited for our purpose since they allow us to look for energy concentration around the dispersion relation, $\omega_{D R}= \pm N \cos \theta$. For the main part of the present study, we will use DNS to calculate $E_{K}(\theta, \omega)$ and $E_{P}(\theta, \omega)$ and check for wave signatures. By employing this spatiotemporal analysis, we have reduced the number of dimensions from 4 to 2 since the outputs are now $2 \mathrm{D}$, in angle-frequency space. This significant reduction in computational cost comes at a small price, however, since the output quantity, $E_{K}(\theta, \omega)$, is an approximated version of the true kinetic energy in $\theta-\omega$ space. This approximation is discussed in Appendix B, where we compare the results of this reduced analysis to those obtained from the full analysis using 4D Fourier transformation.

The procedure outlined in Eqs. (1) and (2) can equally be applied to the velocity fields $\mathbf{u}_{v}$ and $\mathbf{u}_{w}$ obtained after applying the wave-vortex decomposition to our DNS solutions. By doing so, we will arrive at the wave and vortex kinetic energy in angle-frequency space, denoted respectively $E_{w}(\theta, \omega)$ and $E_{v}(\theta, \omega)$. In theory, $E_{w}(\theta, \omega)$ should show all energy being concentrated on the dispersion relation. On the other hand, $E_{v}(\theta, \omega)$ should not have any energy concentration along the dispersion relation, with energy peaking around $\omega \sim \tau^{-1} \approx 0$.

\section{Maintaining the scale dependence}

The reduced analysis described in the previous section reduces all spatial dependence to the orientation of the wave vector with respect to the vertical axis. By doing so, we lose the information relative to the magnitude of the wave vector and hence to the scale of the motions. Since the wave contents of stratified turbulence may change with scale, it is interesting to keep this scale dependence. For example, numerical results reported in Ref. [14] have shown that the waves are energetic mainly at large scales, with less energy being concentrated around the dispersion relation as we move to smaller scales.

In order to maintain the scale dependence, we will consider also the quantity $\mathcal{U}(k, \theta, t)$ which is the sum of $\hat{\mathbf{u}}(\mathbf{k}, t)$ in circular sections of the conical shells in Fig. 1 at fixed $k$. Similarly to 
the reduced analysis above, this sum can then be transformed into frequency space, $\mathcal{U}(k, \theta, t) \Rightarrow$ $\mathcal{U}(k, \theta, \omega)$, and the associated kinetic energy can be formed, denoted $E_{K}(k, \theta, \omega)$. It will hence be possible, using this quantity, to check for signatures of internal gravity waves in stratified turbulence as a function of $k$, i.e., of scale.

\section{B. Wave-vortex decomposition}

The wave-vortex decomposition was introduced by Riley et al. [5] and Lilly [6] as part of a scaling theory in which two distinct sets of reduced equations were obtained in the limit of small Froude number. The first equation, which was found by scaling time using $N^{-1}$, is linear and so should be relevant for the motion of internal gravity waves; its solutions are therefore known as the wave component and they are denoted by $\mathbf{u}_{w}$. The second equation, which was found by scaling time using the eddy turnover time $\tau$, is nonlinear and its solutions are horizontal, $\mathbf{u}_{v}=\mathbf{u}_{v, h}=u_{v, x} \mathbf{e}_{x}+$ $u_{v, y} \mathbf{e}_{y}$; they are known as the vortex component of the flow. In terms of these components, the full velocity field can be written as $\mathbf{u}=\mathbf{u}_{v}+\mathbf{u}_{w}+\mathbf{u}_{s m}$, where $\mathbf{u}_{s m}=\mathbf{u}_{s m}(z)$ corresponds to the shear modes, the horizontally averaged horizontal velocity. In Fourier space, the shear modes correspond to the modes at $k_{h}=0$, i.e., $\hat{u}\left(0,0, k_{z}\right)$ and $\hat{v}\left(0,0, k_{z}\right)$; their particularity within this framework is that they cannot be expressed in terms of the wave-vortex decomposition and so they are part of neither the wave component nor the vortex component. It is convenient to write the wave and vortex components in Fourier space, where they become $\hat{\mathbf{u}}_{v}=\hat{u}_{v} \mathbf{e}_{1}$ and $\hat{\mathbf{u}}_{w}=\hat{u}_{w} \mathbf{e}_{2}$, where $\hat{u}_{v}=$ $\left|\hat{\mathbf{u}}_{v}\right|, \hat{u}_{w}=\left|\hat{\mathbf{u}}_{w}\right|$, and $\mathbf{e}_{1}, \mathbf{e}_{2}$ are unit vectors of the Craya-Herring reference frame $\left(\mathbf{e}_{3}\right.$ is the third unit vector and is $\| \mathbf{k})$. More details on the wave-vortex decomposition and the Craya-Herring reference frame are given in Appendix A. Within the wave-vortex decomposition, one can define the energy of the wave component as $E_{w}=(1 / 2)\left\langle\left|\mathbf{u}_{w}\right|^{2}\right\rangle$ and that of the vortex component as $E_{v}=(1 / 2)\left\langle\left|\mathbf{u}_{v}\right|^{2}\right\rangle$. The notation $\langle\ldots\rangle$ represents a volume average over the numerical domain. If one wishes to account only for the wave and vortex component contributions to the kinetic energy, without considering the shear modes, then a modified form of the kinetic energy becomes $E_{K}^{\prime}=(1 / 2)\left\langle\left|\mathbf{u}_{w}\right|^{2}+\left|\mathbf{u}_{v}\right|^{2}\right\rangle=$ $(1 / 2)\left\langle|\mathbf{u}|^{2}-\left|\mathbf{u}_{s m}\right|^{2}\right\rangle=E_{K}-(1 / 2)\left\langle\left|\mathbf{u}_{s m}\right|^{2}\right\rangle$, where $E_{K}$ is the total kinetic energy. Finally, it is worth pointing out once again that the wave-vortex decomposition is a purely spatial decomposition using which an instantaneous $3 \mathrm{D}$ velocity field is decomposed into a wave and vortex component, without taking into account any information on the temporal evolution of the fields.

\section{NUMERICAL SIMULATIONS}

\section{A. Description of DNS setup}

The DNS simulations presented herein were performed using an in-house code. The code has been used previously for studies of rotating stratified turbulence [32,33]. This code solves the Navier-Stokes equations for an incompressible flow in the presence of buoyancy effects, which are treated using the Boussinesq approximation. In the present setup, buoyancy effects are caused by a linear stratification of the fluid, which is hence characterized by a value of $N$, which is uniform in space and constant in time. The physical constants which are varied are $N$ and the viscosity $v$, while the density diffusivity $\mathcal{D}$ is fixed by our choice of the Prandtl number, $\operatorname{Pr}=v / \mathcal{D}$, which is $\operatorname{Pr}=1$ for all simulations (i.e., $\mathcal{D}=v$ ).

The DNS code is an implementation of a pseudospectral method based on Rogallo's algorithm [34]. The numerical domain is a cube with periodic boundary conditions. The grid spacing is uniform in all three directions and the number of grid points is $N_{x}=N_{y}=N_{z}$. The dimensions of the domain are $L_{x}=L_{y}=L_{z}=2 \pi$. Dealiasing of the quadratic terms is obtained by the combination of phase shifting and truncation of the modes with highest $k\left[k>k_{\max }=(2 \sqrt{2} / 3) k_{\mathrm{nyq}}\right.$, where $k_{\text {nyq }}=N_{x} / 2$ is the Nyquist wave number]. The viscous and diffusive terms are treated exactly using integrating factors. The numerical time integration is performed using a third-order AdamsBashforth method. The grid spacing is chosen to ensure accurate resolution of the small scales during steady state. For this we use the condition $k_{\max } \eta \approx 1$, where $\eta=\left(v^{3} / \epsilon\right)^{1 / 4}$ is the Kolmogorov 
length scale and $k_{\max }$, defined above, is the maximum resolved wave number. More details on the set of simulations reported in this paper are given in Sec. III C.

\section{B. Forcing technique}

In the present study, forcing is required in order to obtain a statistically stationary solution for a period of time. Over this period, it is assumed that the waves within the stratified turbulence are also in statistical stationarity so that we can extract their amplitude computing a time Fourier transform. One of the most common forcing schemes used in previous numerical studies has been vortical forcing, which is directed along the $\mathbf{e}_{1}$ direction and so excites only the vortex component of the flow. Vortical forcing has often been implemented so as to force only the modes with $k_{z}=0$, which should generate vertically elongated structures that subsequently go unstable, thus injecting energy in the turbulent flow [3,13,35-37]. A number of studies have employed other schemes, such as forcing the wave component [14,38] or classical isotropic forcing on spherical shells in Fourier space [39-41]. For our present investigation of waves in stratified turbulence, we seek a different forcing scheme in which wave and vortex components are excited equally so that the prevalence of one or the other component should be determined solely by the dynamics. In addition, we choose not to force isotropically and instead to use an anisotropic forcing, which is "pancake"-like and so qualitatively similar to the structure of stratified turbulence. This choice is partially motivated by the recent work of Kunze [42], who reported that in the ocean energy is injected into the submesoscale motions $\left(\ell_{h}<1 \mathrm{~km}\right)$ by an anisotropic breaking of internal gravity waves, which could thereby feed stratified turbulence with its highly anisotropic dynamics over the submesoscales.

In order to satisfy the above conditions, we devised another forcing technique which we term cylindricalforcing. It consists in forcing the modes in cylindrical shells, whose axis is the $k_{z}$ axis and who are characterized by a radius $k_{h, f}$ and are of finite vertical extent, from $k_{v, f \min }$ to $k_{v, f \max }$. The two cylindrical shells (one at positive $k_{z}$ and one at negative $k_{z}$ ) on which the forcing is concentrated are schematically depicted in Fig. 2. The force $\hat{\mathbf{f}}$ within the cylindrical shells has components $\hat{f}_{1}$ and $\hat{f}_{2}$ in the Craya-Herring frame, which are chosen to be of equal modulus, on average, so that wave and vortex component of the flow are excited in equal proportions (note that $\hat{f}_{3}=0$ since $\mathbf{k} \cdot \hat{\mathbf{f}}=0$ to satisfy incompressibility). An advantage of this forcing with respect to an isotropic one is that cylindrical forcing does not directly excite the shear modes, which lie on the $k_{z}$ axis. It is well known that shear modes grow uncontrollably in simulations of forced stratified turbulence [9,37], so this choice limits their growth. In explicit form, the implemented cylindrical forcing is given by

$$
\hat{f_{1}}=x_{F} e^{i \theta_{1}} \cos \phi, \quad \hat{f}_{2}=x_{F} e^{i \theta_{2}} \sin \phi
$$

for $k_{h, f}-\Delta k / 2<k_{h}<k_{h, f}+\Delta k / 2$ and $k_{v, f \text { min }} \leqslant\left|k_{z}\right| \leqslant k_{v, f \max }$, and $\hat{\mathbf{f}}=0$ otherwise. Here $\Delta k=1$ is the wave-number increment and $\theta_{1}, \theta_{2}$, and $\phi$ are uniformly distributed random numbers between 0 and $2 \pi$. As a result, the forcing is uncorrelated in time (white noise in time). The value of the forcing amplitude $x_{F}$ is computed at each time step using the constant power minimal forcing scheme [16], which ensures that the power injected is fixed at a chosen value $P^{*}$ while keeping the forcing magnitude $|\hat{\mathbf{f}}|=\left|x_{F}\right|$ at a minimum. A constant power forcing allows us to know the total dissipation rate $\epsilon+\epsilon_{p}$ during steady state, since $\epsilon+\epsilon_{p}=P^{*}$ once steady state is reached. Here $\epsilon_{p}$ is the potential energy dissipation rate. With a reasonable choice for the mixing coefficient, $\Gamma=\epsilon_{p} / \epsilon$, in the SST regime (see, e.g., Ref. [37]), this allows us to obtain an estimate of the value of $\operatorname{Re}_{b}$ during steady state. This ability is helpful in selecting an appropriate value of $P^{*}$ given a desired value of $\operatorname{Re}_{b}$ at steady state.

For the present simulations, we have chosen $k_{h, f}=3, k_{v, f \min }=8$, and $k_{v, f \max }=12$, which correspond to a pancake-like force $f$ with typical aspect ratios between $8 / 3 \approx 2.67$ and 4 . A realization of $\mathbf{f}$ on a $256^{3}$ grid has been computed and Fig. 3 shows $f_{x}$ on a vertical $x-z$ slice. Note that, as will be shown subsequently, the number of layers in the velocity and density fields is not given by the number of layers in Fig. 3. This confirms that the vertical length scale is chosen by the dynamics of the flow; in particular it will be such that the vertical Froude number is of order 


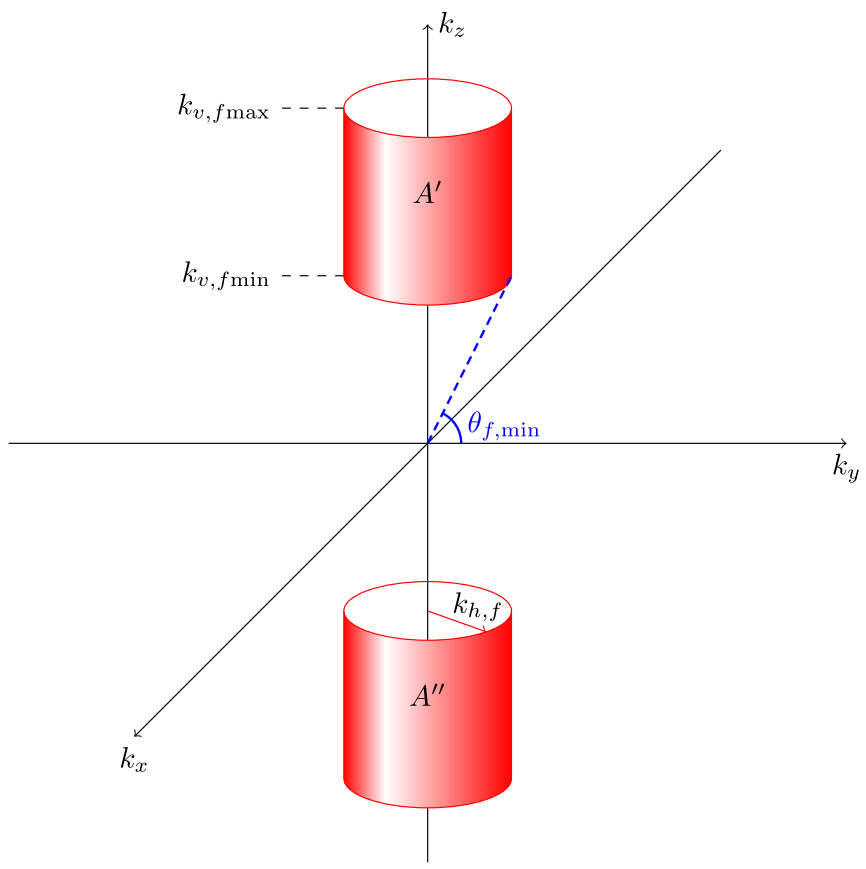

FIG. 2. Illustration of cylindrical forcing. The force $\hat{\mathbf{f}}$ is applied in Fourier space and is active only on the cylinder areas, $A^{\prime}$ and $A^{\prime \prime}$, which in a discrete formulation become cylindrical shells. The forcing is active in an interval of $\theta$; the minimum value of $\theta$ that is forced is shown.

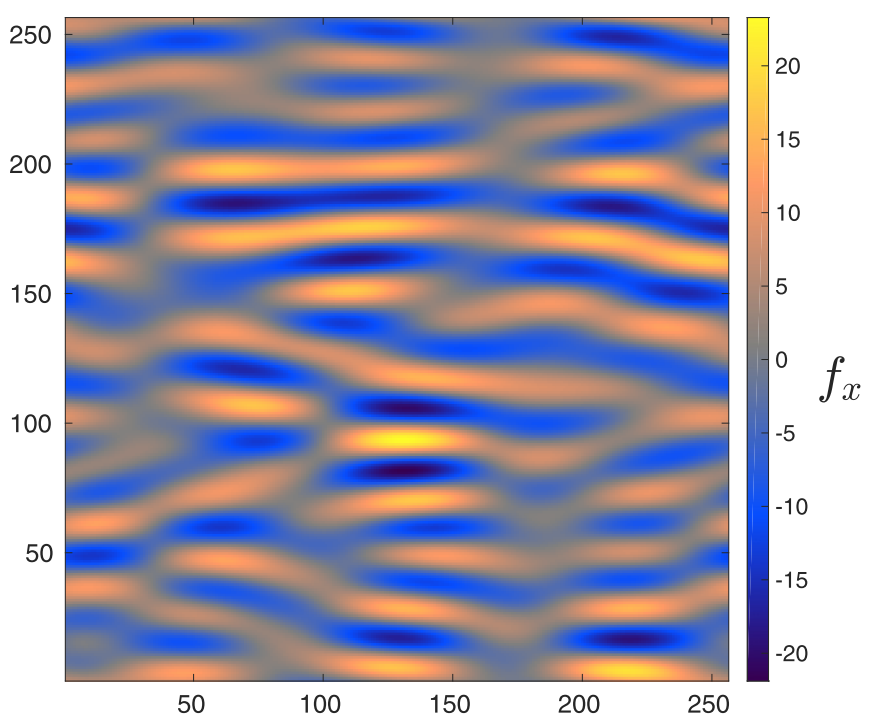

FIG. 3. Realization of cylindrical forcing with $k_{h, f}=3, k_{v, f \min }=8, k_{v, f \max }=12$ on a $256^{3}$ grid. Visualization of $f_{x}$ on an $x-z$ plane (the units of $f_{x}$ are arbitrary). 
TABLE I. List of numerical parameters and nondimensional quantities during steady state for the DNS simulations of this study. The naming convention of the runs is based on their value of $\operatorname{Re}_{b}$.

\begin{tabular}{cccccccccc}
\hline \hline Run & Grid & $\operatorname{Re}_{b}$ & $\mathrm{Fr}_{h}$ & $\operatorname{Re}$ & $k_{\max } \eta$ & $M$ & $1 /\left(\omega_{\min } \tau\right)$ & $\omega_{\max } / N$ & $\omega_{\max } T_{K}$ \\
\hline R1.6 & $512^{3}$ & 1.6 & 0.032 & 1560 & 0.99 & 256 & 3.2 & 1.28 & 1.02 \\
$\mathrm{R} 3.8$ & $1024^{3}$ & 3.8 & 0.030 & 4360 & 0.99 & 256 & 3.0 & 1.28 & 0.65 \\
$\mathrm{R} 9.9$ & $2048^{3}$ & 9.9 & 0.029 & 11800 & 0.99 & 192 & 0.72 & 3.88 & 1.23 \\
\hline \hline
\end{tabular}

unity once we approach the SST regime. The vertical Froude number is defined as $\mathrm{Fr}_{v}=u_{h} / N \ell_{v}$, where $\ell_{v}$ is the vertical length scale of the flow, and $\mathrm{Fr}_{v} \sim 1$ is one of the central results of the scaling analysis for the SST regime $[11,13]$.

\section{Details of numerical simulations}

A total of three DNS simulations were performed as part of this study. This set of DNS runs is at constant stratification $N$ but with a decreasing viscosity $v$, in order to increase the buoyancy Reynolds number from $\operatorname{Re}_{b} \approx 1$ to $\operatorname{Re}_{b} \approx 10$. As a result, the resolution of the simulations is increased, from $512^{3}$ grid points in the first run, to $1024^{3}$, to $2048^{3}$ in the final run. The injected power is kept constant at $P^{*}=1$. The Froude number is approximately constant across the three runs, at $\mathrm{Fr}_{h} \approx 0.03$. We here define the Froude number, which is a horizontal Froude number based on $\ell_{h}$, as $\operatorname{Fr}_{h}=\epsilon / N u_{h}^{2}$, taking advantage of the relationship $\epsilon \sim u_{h}^{3} / \ell_{h}$ [12]. In this form, the Froude number represents a ratio between the timescale of energy dissipation $\tau=u_{h}^{2} / \epsilon$ (recall that $u_{h}^{2}$ dominates the kinetic energy in SST) and the buoyancy timescale $N^{-1}$. The Reynolds number is similarly defined as $\operatorname{Re}=u_{h}^{4} / v \epsilon$. In what follows, the above definition of $\tau$ will be used to estimate the eddy turnover time. The horizontal velocity scale is defined as $u_{h}=\sqrt{(1 / 2)\left\langle u^{2}+v^{2}-\left|\mathbf{u}_{s m}\right|^{2}\right\rangle}$, where $(1 / 2)\left\langle\left|\mathbf{u}_{s m}\right|^{2}\right\rangle$ is the kinetic energy of the shear modes and $u, v$ are the horizontal components of the full velocity field, $\mathbf{u}=[u, v, w]$. In this definition of $u_{h}$, we have removed the contribution of the shear modes because these are large-scale motions that are fed by a slow but continuous upscale transfer of energy, which tends to accumulate energy in these modes. Despite this transfer being limited by our choice of cylindrical forcing, the long integration times necessary to reach steady state with this forcing scheme lead to the shear modes dominating the kinetic energy during the steady-state period, as will be shown in the next section. A more representative value of $\mathrm{Fr}_{h}$ during steady state is obtained by subtracting their energy from the horizontal kinetic energy, as done in Ref. [41].

A full list of nondimensional parameters and important quantities for each simulation is given in Table I. Note that the values given in Table I, as well as the approximate values for $\operatorname{Re}_{b}$ and $\mathrm{Fr}_{h}$ given above, are computed from time-averaged physical quantities, such as $\bar{\epsilon}$ and $\overline{u_{h}^{2}}$, where the time averages, $\overline{(\ldots)}$, are carried out over the statistically stationary period of each run. Runs R1.6 and R3.8 are initialized with a weakly energetic artificial isotropic velocity field with random phases and with zero density perturbations throughout the numerical domain. To save computational time in the case of the highest resolution simulation, R9.9, this run was initialized with the final snapshot of the R3.8 run.

For the spatiotemporal analysis, the time series of $\mathcal{U}(\theta)$ and $\mathcal{A}(\theta)$ are accumulated over a period $T$ during steady state. This period defines the minimum frequency of our analysis as $\omega_{\min }=2 \pi / T$. The total number of samples per time series is $M$ and the time interval between successive samples is $T_{\text {sample }}$. From $T_{\text {sample, }}$, we can calculate the maximum frequency of a signal that we are able to successfully reconstruct (the Nyquist frequency), $\omega_{\max }=(1 / 2) 2 \pi / T_{\text {sample }}=\pi / T_{\text {sample }}$. For us to be able to capture the highest frequencies of internal gravity waves in our simulations, it is necessary that $\omega_{\max } \geqslant N$. On the other end of the spectrum, we expect the lowest frequencies to be those of quasihorizontal waves with the same aspect ratio of the vortices; such waves have $\omega \sim \tau^{-1}$ in 


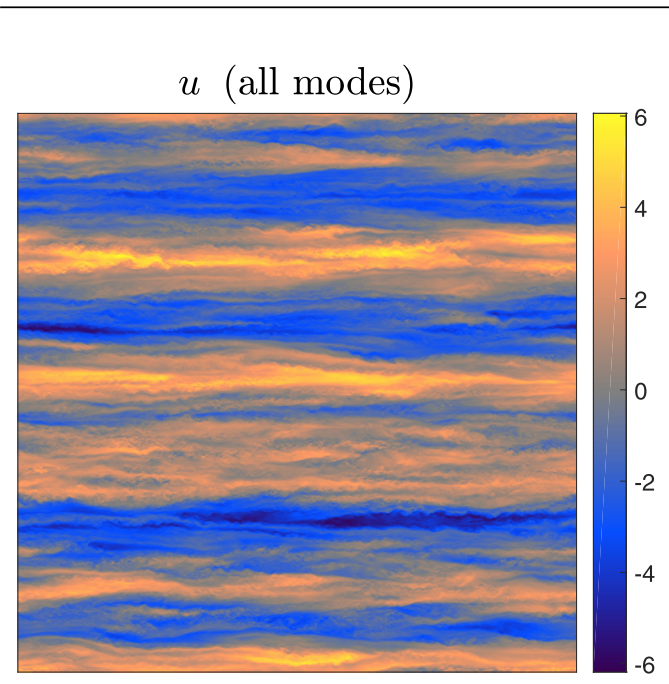

(a)

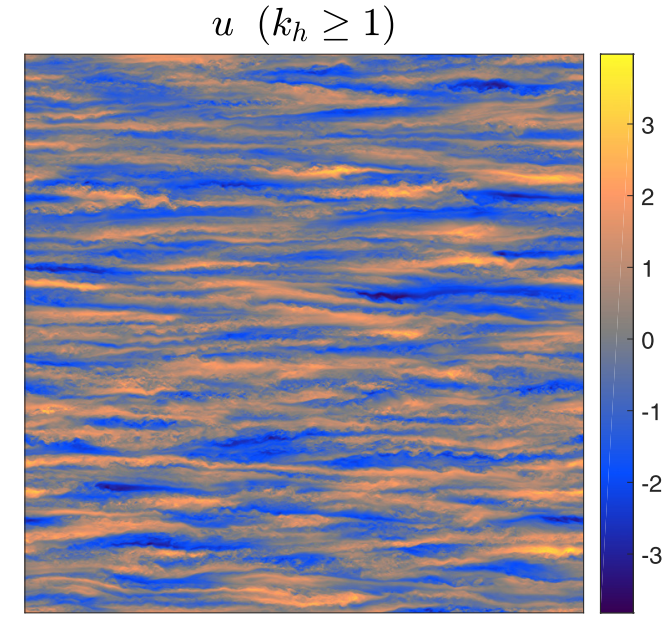

(b)

FIG. 4. Visualization of $x$ component of velocity on the central $x-z$ plane in run R9.9 at the last time instant of the simulation: (a) $u$ and (b) $u$ minus its horizontal average over the whole domain, i.e., keeping only the contributions of modes with $k_{h} \geqslant 1$ and removing the shear modes at $k_{h}=0$.

SST [11]. As a result, we have chosen the lowest frequency of our spatiotemporal analysis so that $1 / \omega_{\min } \tau \gtrsim 1$. The specific values used in our spatiotemporal analyses are given in Table I. The value of $\omega_{\max } T_{K}$ is also given, where $T_{K}=(v / \epsilon)^{1 / 2}$ is the Kolmogorov timescale; this quantity is the temporal equivalent of $k_{\max } \eta$ and should therefore be $\omega_{\max } T_{K} \gtrsim 1$. Note that, in order to obtain the $\omega_{\min }$ values in Table I, very long time integrations of the DNS simulations have been carried out. Indeed, for runs R1.6 and R3.8, the period $T$ is equivalent to more than $18 \tau$ (or $\approx 100$ buoyancy periods $\left.T_{B}, T_{B}=2 \pi / N\right)$ and, for run $\mathrm{R} 9.9, T$ is about $4.5 \tau\left(\right.$ or $\left.\approx 25 T_{B}\right)$.

\section{RESULTS}

\section{A. Visualizations}

We begin the section on the DNS results by showing visualizations of the velocity and density fields during steady state. In Figs. 4 and 5, visualizations of $u$ (full $x$ component of velocity and this same component with the shear modes removed) and $\rho^{\prime} g / \rho_{0}$ are given on a vertical slice at the final time instant of the steady state period of run R9.9. From Fig. 4, it is clear that $u$ is dominated by shear modes with a relatively large vertical length scale. It can be seen from the $u$ field with shear modes removed that, superimposed on the shear modes, layered structures with smaller horizontal and vertical length scales are present, together with even smaller scale features. If we now turn to the visualization of the density field in Fig. 5, we observe similar layered structures and the frequent manifestation of billows that are highly reminiscent of those associated with shear instabilities such as the Kelvin-Helmholtz instability (note that since the shear modes consist in horizontal flow they do not create density perturbations and so are not visible in Fig. 5). The overall picture of this turbulent flow is consistent with the description of SST, with layered structures that are vertically sheared by one another, leading to shear instability and small-scale turbulence $[13,43]$. It is also worth emphasizing that the vertical length scales in the $u$ and $b$ fields do not appear to be set by the vertical length scale of the forcing; cf. Fig. 3. Specifically, the layered structures in Figs. 4(b) and 5 have a range of vertical length scales, perhaps including the forcing length scale, yet there appears to be a large number of "thin" energetic structures with vertical scale smaller than the forcing scale. Indeed, the average vertical integral length scale of the $u$ and $v$ fields with shear modes removed 


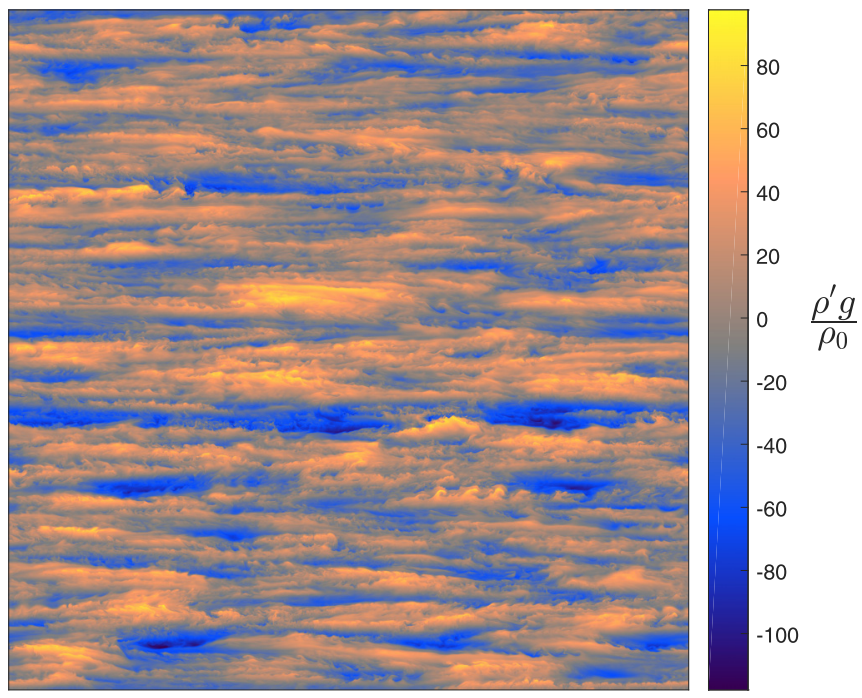

FIG. 5. Visualization of $\rho^{\prime} g / \rho_{0}$ in run R9.9 (same plane and time instant as in Fig. 4).

[as in Fig. 4(b)], calculated using a standard definition [44], is $\ell_{v} \approx 0.056$ at this time instant. This value of $\ell_{v}$ gives a vertical Froude number $\mathrm{Fr}_{v} \approx 0.60$, which is order 1 , as expected for SST. On the other hand, the integral length scale of the forcing realization shown in Fig. 3 is $\approx 0.099$ (note that the side of the cubic domain has length $2 \pi$ ). The integral length scale may not be the best method to estimate the scale of the forcing, which consists in the sum of a limited number of modes, but we use it for consistency. We argue that this difference in the value of $\ell_{v}$ together with the visual evidence from Figs. 3, 4(b), and 5 are sufficient to show that the flow largely self-organized to arrive at this vertical structure. We can also see from Fig. 4(a) that in the case of the shear modes they have a larger vertical scale than the forcing, again showing that the forcing does not directly impart its vertical scale on the flow.

\section{B. Time evolution and stationarity}

The time evolution of quantities such as kinetic energy starting from the beginning of the simulations until statistical stationarity is now considered. Figure 6(a) is a plot of the time evolution of the volume-averaged kinetic energy, $E_{K}$, of the kinetic energy excluding the shear modes, $E_{K}^{\prime}$, and of $u_{h}^{2}$ for run R3.8. Time is nondimensionalized by the steady-state time average of the eddy turnover time, $\tau$ (i.e., $\overline{u_{h}^{2}} / \bar{\epsilon}$ ). The first thing to note from Fig. 6(a) is that $E_{K}$ grows continuously over time and does not reach stationarity because of the shear modes that continue to grow in energy content. On the other hand, $E_{K}^{\prime}$ does reach statistical stationarity, showing that all the other modes do reach a steady state. However, $E_{K}^{\prime}$ is significantly lower than $E_{K}$ at steady state, confirming that the shear modes dominate the kinetic energy at this stage, containing almost $80 \%$ of the overall kinetic energy. In addition, $u_{h}^{2}$ is only slightly lower than $E_{K}^{\prime}$, which means that the vertical velocity accounts for a small fraction of the kinetic energy and that the flow is quasihorizontal at the large, energy-containing scales.

Next, we apply the wave-vortex decomposition to the velocity field and consider the kinetic energy in the wave and vortex components, $E_{w}$ and $E_{v}$, whose sum is equal to $E_{K}^{\prime}$. The evolution of $E_{w}, E_{v}, E_{K}^{\prime}$, and of the potential energy, $E_{P}$, over the course of run R3.8, is given in Fig. 6(b). This plot shows that after the transient period the wave and vortex kinetic energy both reach steady state, with a larger kinetic energy in the vortex component compared to the wave component. This happens despite the fact that both components are equally forced, which goes to show that the dynamics acts 


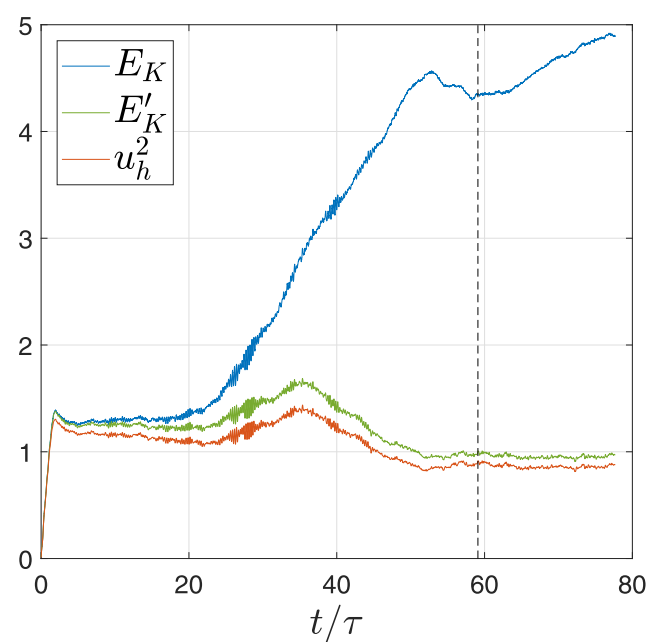

(a)

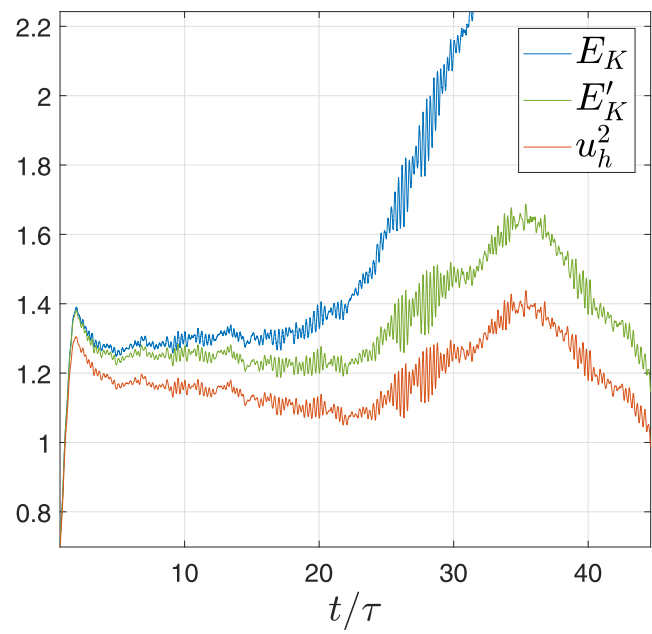

(c)

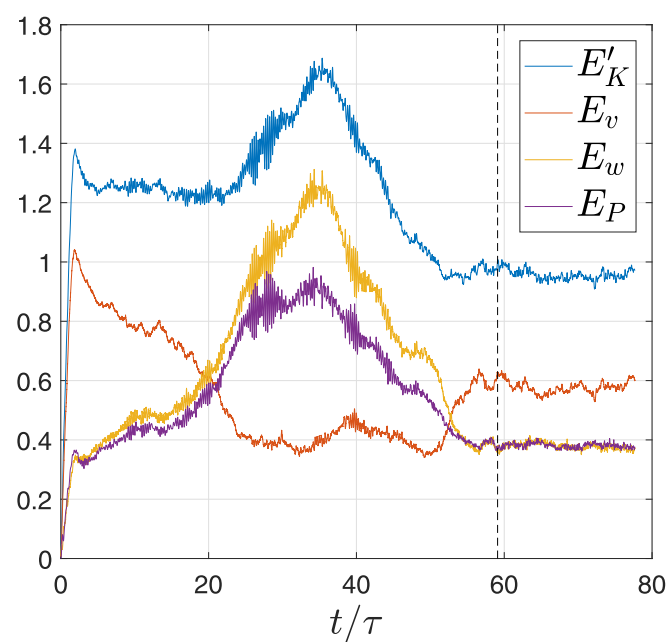

(b)

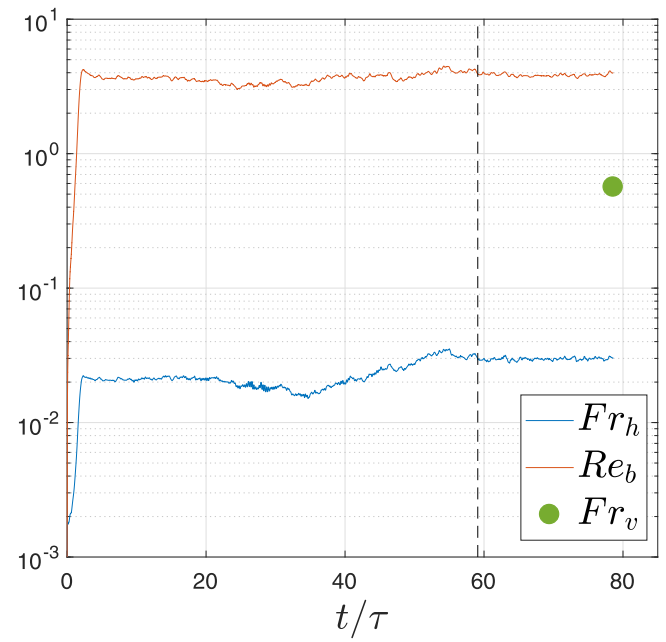

(d)

FIG. 6. Time evolution of volume-averaged quantities in run R3.8. (a) Kinetic energy evolution: $E_{K}, E_{K}^{\prime}$, and $u_{h}^{2}$; (b) evolution of $E_{K}^{\prime}, E_{P}$, and wave and vortex kinetic energies $E_{w}$ and $E_{v}$; (c) enlargement of panel (a), showing the initial transient period; (d) evolution of $\mathrm{Fr}_{h}$ and $\mathrm{Re}_{b}$ over time and vertical Froude number $\mathrm{Fr}_{v}$ shown at the last time step of the run. The dashed lines in panels (a), (b), and (d) show the time at which the steady state is considered to begin.

to increase $E_{v}$ over $E_{w}$. Having said this, the difference between $E_{v}$ and $E_{w}$ during steady state is contained, as these quantities remain of the same order of magnitude. Another interesting point is that the potential energy has a similar evolution to the kinetic energy of the wave component and that, during steady state, $E_{P} \approx E_{w}$. This may be a manifestation of equipartition of kinetic and potential energy in the internal gravity waves in this simulation. It may also be due to overturning motions, such as the billows seen in Fig. 5, which are similarly characterized by equipartition (in this case in an approximate way only) between kinetic and potential energies. Indeed, as discussed in previous work on stratified turbulence [36], the billows have a poloidal velocity field, resulting in their energy being spuriously added to the wave component. On the other hand, the vortex component $\mathbf{u}_{v}$ represents a purely toroidal velocity field. 
From Figs. 6(a) and 6(b), it is clear that the simulation with cylindrical forcing has an extended transient period before reaching statistical stationarity after a little more than 50 eddy turnover times. The details of this transient evolution are considered in Fig. 6(c), which is an enlargement of the early times of Fig. 6(a). Oscillations of the kinetic energy are clearly visible, and they appear in "bursts" of varying amplitude. The period of the oscillations seems to be approximately constant and, by counting the peaks in a number of bursts, it is estimated to be $T \approx 0.26 \tau \approx 1.4 T_{B}$. This results in a frequency of the oscillations of $\approx 0.71 N$. Considering that these are oscillations on the kinetic energy which contain quadratic terms- $u^{2}, v^{2}$, and $w^{2}$-if the oscillations are created by waves with $u$ component $u \propto \exp [i(\mathbf{k} \cdot \mathbf{x}-\omega t)]$, then we have $u^{2} \propto \exp [2 i(\mathbf{k} \cdot \mathbf{x}-\omega t)]=$ $\exp [i(2 \mathbf{k} \cdot \mathbf{x}-2 \omega t)]$ and the frequency of these oscillations is $2 \omega$, at least in a simplistic sense. This results in $\omega \approx 0.36 N$. This frequency is consistent with that of waves with angle $\theta$ corresponding to the lowest angle excited by the forcing, $\theta_{f, \min }=\tan ^{-1}\left(k_{v, f \min } / k_{h, f}\right)=\tan ^{-1}(8 / 3) \approx 69.4^{\circ}$, which corresponds to a wave frequency $\omega=N \cos \theta_{f \text {, min }} \approx 0.35 N$. Hence, the transient period is characterized by significant changes of both kinetic and potential energy in the presence of waves that are excited by the forcing and that appear in nonstationary bursts, whose amplitude initially grows and then decays. Once the wave bursts cease, around $t=45 \tau$, the kinetic and potential energy begin to relax toward their statistically stationary values.

The evolution of nondimensional quantities is considered in Fig. 6(d), showing the horizontal Froude number and the buoyancy Reynolds number over the course of the simulation. The value of $\mathrm{Fr}_{h}$ remains low during the entire evolution of the simulation, including the transitory state, before reaching its steady state value, $\mathrm{Fr}_{h}=0.03$. Similarly, $\mathrm{Re}_{b}$ rapidly evolves from a low value to a value above unity before finally settling to its stationary value. The vertical Froude number, $\mathrm{Fr}_{v}=u_{h} / N \ell_{v}$, which, in our definitions, is the only nondimensional number containing a length scale, is also shown but only at the final time step of the run, where $\mathrm{Fr}_{v}=0.56$. The reason for this is that the calculation of the vertical length scale $\ell_{v}$ is a numerically intensive postprocessing computation involving the full velocity field (specifically its components $u$ and $v$ ). Nonetheless, Fig. 6(d) highlights that $\mathrm{Fr}_{v}$ has a value of order unity during steady state that is over an order of magnitude larger than the value of $\mathrm{Fr}_{h}$, confirming the high anisotropy of the flow.

\section{Spatiotemporal analysis}

\section{Evidence of the dispersion relation}

One of the first questions that one can answer using the spatiotemporal analysis is whether energy in the $\theta-\omega$ space concentrates around the dispersion relation. This would be a clear signature of internal gravity waves. To check this possibility, color maps of $E_{K}(\theta, \omega)$ and of $E_{P}(\theta, \omega)$ for all three runs are given in Fig. 7. These quantities are computed over the steady-state period of each run. In the case of run R9.9, $E_{P}(\theta, \omega)$ calculated using Eqs. (1) and (2) is not available. Instead, the quantity that is given in Fig. 7(f) is $E_{P}^{\prime}(\theta, \omega)=\sum_{k} E_{P}(k, \theta, \omega)$. As discussed in Appendix B, $E_{P}(\theta, \omega)$ and $E_{P}^{\prime}(\theta, \omega)$ are similar but not identical quantities. As can be seen in Fig. 7, the energy does concentrate around the dispersion relation, showing that internal gravity waves are an important feature of the DNS. By further inspecting Figs. 7(a) and 7(b), where the dispersion relation is plotted, we can observe that the frequencies contained in the DNS are systematically slightly higher than $\omega_{D R}$ but globally they follow the dispersion relation convincingly (these observations are true also for the results of the other DNS runs presented in Fig. 7). There is a signature of waves from low frequency, $\omega \approx 0$, to high frequency, $\omega \approx N$. Therefore, waves covering practically the entire allowable frequency spectrum, $0 \leqslant|\omega| \leqslant N$, are present during the steady-state period of the DNS. This means that not only low-frequency waves with $\omega \sim \tau^{-1} \ll N$ are observed, as suggested in Ref. [11], but also higher frequency waves with $\omega \sim N$ are present in our DNS. This finding confirms and extends the observation of waves with $\omega \sim N$ in homogeneous stratified turbulence in Ref. [14] for a number of individual wave vectors in their simulations.

In the color maps of $E_{K}(\theta, \omega)$, there are energetic regions within the range $\theta_{f, \min }<|\theta|<\pi / 2$ and at low frequency, where the dispersion relation is not visible and the energy is distributed close 


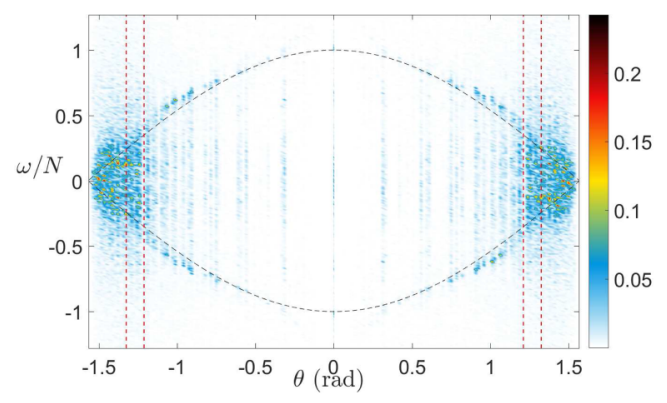

(a)

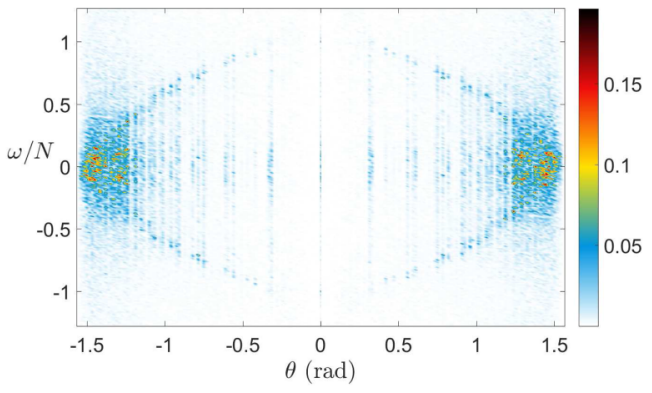

(c)

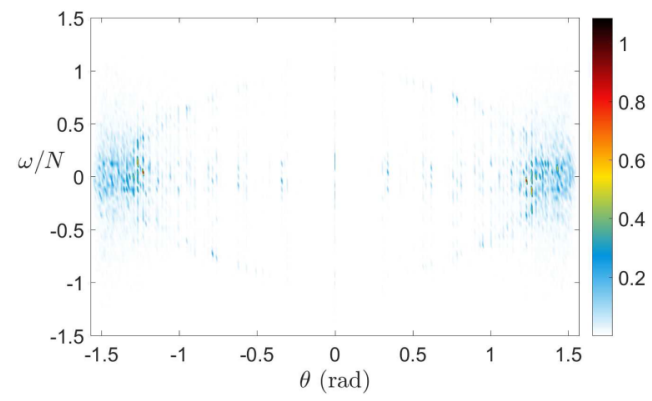

(e)

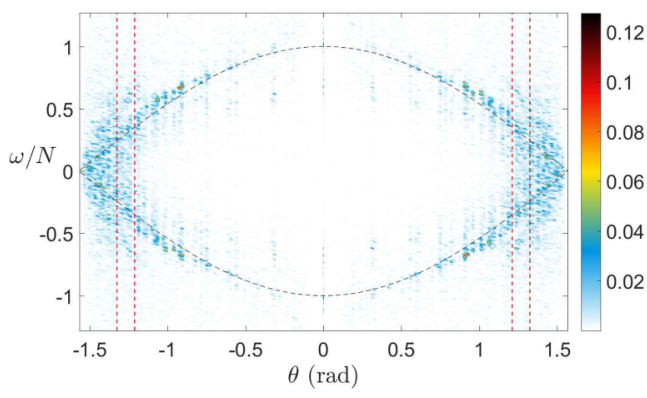

(b)

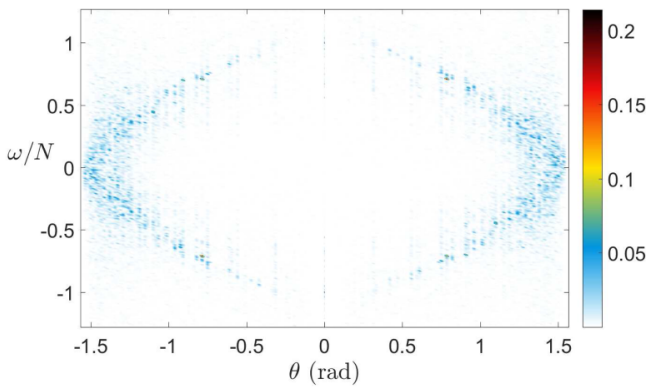

(d)

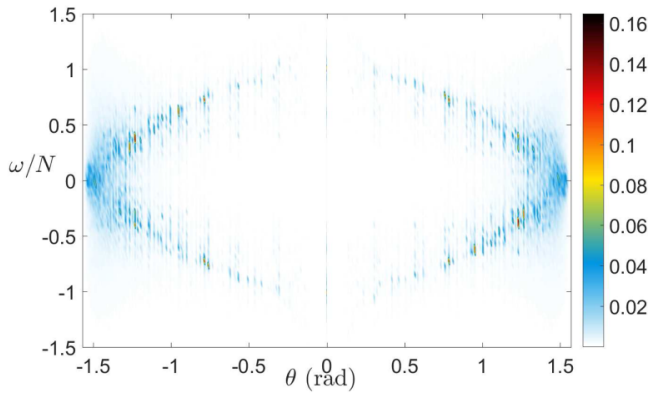

(f)

FIG. 7. Color maps of $E_{K}(\theta, \omega)$ and $E_{P}(\theta, \omega)$. Left column [(a), (c), (e)] $E_{K}(\theta, \omega)$. Right column: [(b), (d)] $E_{P}(\theta, \omega)$ and (f) $E_{P}^{\prime}(\theta, \omega)$. [(a), (b)] Run R1.6; [(c), (d)] run R3.8; [(e), (f)] run R9.9. In panels (a) and (b), the black dashed curves show the dispersion relation of internal gravity waves, $\omega / N= \pm \cos \theta$, while the red dashed lines show the minimum and maximum forcing angles, $\theta= \pm \theta_{f \text {,min }}$ and $\theta= \pm \theta_{f \text {, max }}$. The shear modes at $\theta=\pi / 2$ are not plotted because their high intensity concentrated in a single point $(\omega=0)$ makes all other points undistinguishable. Note that the color maps have a central symmetry with respect to $(\theta, \omega)=(0,0)$ because they are constructed from real data.

to uniformly over a frequency and angular range. These regions correspond to wave vectors that are almost vertical and therefore to quasihorizontal structures in physical space. In other words, they correspond to the layers of stratified turbulence, whose dynamics probably consists of a combination of waves and vortices that interact nonlinearly. In the plots of $E_{P}(\theta, \omega)$, the dispersion relation remains distinguishable as we move toward high absolute values of $\theta$. Also here the energy becomes more distributed over neighboring frequencies, including $\omega=0$. Returning to $E_{K}(\theta, \omega)$, 


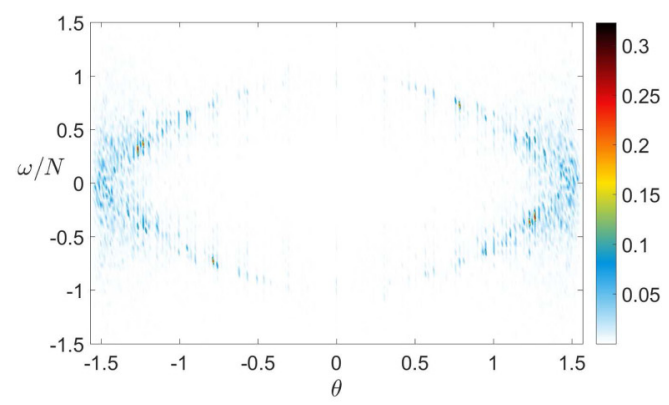

(a)

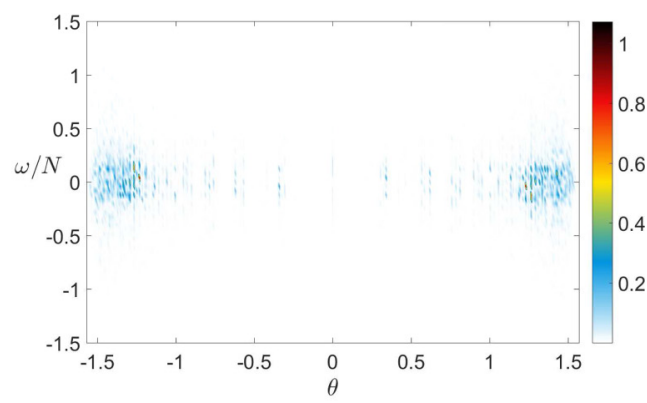

(b)

FIG. 8. Spatiotemporal analysis of wave and vortex components in run R9.9: (a) $E_{w}(\theta, \omega)$; (b) $E_{v}(\theta, \omega)$.

its concentration is over two regions beginning at the lowest forcing angle, $\pm \theta_{f \text {, min }}$, and extending up to $\theta= \pm \pi / 2$, well beyond the maximum forcing angle $\theta_{f, \max }=\tan ^{-1}\left(k_{v, f \max } / k_{h, f}\right) \approx 1.33 \mathrm{rad}$. This is evidence that there is a continuous transfer of kinetic energy from wave vectors at the forcing angles toward higher close-to-vertical angles. The energy goes from the mildly anisotropic structures of the forcing toward the highly anisotropic structures of stratified turbulence in a regime close to SST, as shown in Figs. 4 and 5. Both kinetic and potential energy plots show that there is also some energy that gets transferred to angles smaller than $\theta_{f \text {, min }}$, mostly in the form of waves with $\omega \sim N$. The boundary between these two dynamical regions appears to be $\theta_{f, \text { min }}$ and so the forcing may have an influence on the precise energy distribution in $\theta$ - $\omega$ space. Notwithstanding, the presence of high-frequency waves with $\omega \sim N$ and low-frequency anisotropic structures should be a general result of this stratified turbulence regime.

\section{Spatiotemporal analysis of wave and vortex components}

In the case of run R9.9, a more detailed analysis has been carried out, including a spatiotemporal analysis of the wave and vortex components. The outputs are the wave and vortex kinetic energy in $\theta-\omega$ space, $E_{w}(\theta, \omega)$ and $E_{v}(\theta, \omega)$ respectively, computed following the procedure of Sec. II A 1. These quantities allow one to inspect whether the dispersion relation is entirely contained in $E_{w}(\theta, \omega)$ or if it is also visible in $E_{v}(\theta, \omega)$, for example, because of nonhorizontal isopycnals. Indeed, as discussed in a theoretical and numerical study of the wave-vortex decomposition [45], the decomposition should be modified if the isopycnals are not flat and present significant deviations. In this case, the vortex component $\mathbf{u}_{v}$ should not be a horizontal velocity as in the classical decomposition but should actually represent a flow along the isopycnal surfaces. In their simulation of a mixing layer, an oscillatory behavior was observed on time series of $E_{v}$, which was ascribed to waves made visible in the vortex component by the large deviations of the isopycnals associated with an isolated shear instability [45].

The quantities $E_{w}(\theta, \omega)$ and $E_{v}(\theta, \omega)$ are depicted in Fig. 8. The wave kinetic energy in Fig. 8(a) presents a clear signature of internal gravity waves as it appears that most of the energy is on or in the direct surroundings of the dispersion relation. On the other hand, Fig. 8(b) showing the vortex kinetic energy, contains no evidence of the dispersion relation. It therefore can be said that the wave-vortex decomposition does a relatively good job in decomposing the kinetic energy of this simulation, at least from the information that can be obtained from the color maps in Fig. 8. The only "gray area" is in the region close to $\theta= \pm \pi / 2$, where the dispersion relation shown by $E_{w}(\theta, \omega)$ appears more diffused as energy is "smeared" both toward frequencies higher than $\omega_{D R}$ and lower than $\omega_{D R}$, around $\omega \approx 0$. This result is somewhat expected considering that the low wave frequencies in these regions mean that there is not a significant separation between the timescales $2 \pi / \omega_{D R}$ and $\tau$ so that the wave-vortex decomposition should break down. Conversely, the decomposition into motions that obey the dispersion relation in $E_{w}(\theta, \omega)$ and motions at $\omega \approx 0$ in $E_{v}(\theta, \omega)$ is most successful 
for $\theta$ different from $\theta \approx \pm \pi / 2$, where we recover a clear timescale separation between waves and vortices.

Overall, the results show that, at least qualitatively, the wave-vortex decomposition works well, especially for angles $-\theta_{f, \text { min }}<\theta<\theta_{f \text {, min }}$. At larger angles, $|\theta|>\theta_{f, \text { min }}$, as we approach $\theta= \pm \pi / 2$, the results become less clear cut: There is a hint of the dispersion relation in $E_{w}(\theta, \omega)$ but both wave and vortex kinetic energies are relatively diffuse and are spread over overlapping regions of the $(\theta, \omega)$ plane. Finally, the differences between these results, which do not show sign of the dispersion relation in the vortex component, and those of an isolated shear layer [45] are not fully understood. Indeed, the presence of shear instabilities in this run, R9.9 (see Fig. 5), suggests substantial isopycnal displacements, albeit at relatively small, and not very energetic, scales.

\section{Wave content as a function of scale}

For the high-resolution run, R9.9, we have also carried out the scale-dependent spatiotemporal analysis, which yielded the quantities $E_{K}(k, \theta, \omega)$ and $E_{P}(k, \theta, \omega)$. In practice, this allows us to consider maps in $(\theta, \omega)$ coordinates similar to those in Fig. 7 for every wave number $k$. With this detailed information, we can check if the wave content of the DNS run is mainly at large scales, similar to what was observed in previous simulations of stratified turbulence [14]. It also means we are able to investigate possible Doppler shifts of the wave frequencies, which depend on the specific scale of the waves, as quantified by $k$.

By careful inspection of $E_{K}(k, \theta, \omega)$ and $E_{P}(k, \theta, \omega)$, we have ascertained that most of the wave signal at angles smaller than the forcing angles, i.e., $|\theta|<\theta_{f, \min }$, is at the largest scales of the simulation up to approximately $k=8$. The smallest wave number excited by the forcing is $k=$ 9; i.e., it is given by $\sqrt{k_{h, f}^{2}+k_{v, f \text { min }}^{2}}=\sqrt{3^{2}+8^{2}} \approx 8.54=9$ since the integer wave-number bins contain wave numbers between $k-0.5$ and $k+0.5$. This means that, at these $\theta$, most of the energy in the waves is contained in scales larger than the forcing scales. We analyze only the energy content in the waves with $|\theta|<\theta_{f \text {,min }}$ because in this region the wave signal is clearest; for higher angles, $|\theta|>\theta_{f, \min }$, the dispersion relation is less distinguishable and the separation between waves and vortices in the $(\theta, \omega)$ plane is not as clear cut (even when using the wave-vortex decomposition; see Fig. 8). We therefore prefer not to make any statements about the wave energy (or the vortex energy) in this region of the $(\theta, \omega)$ plane corresponding to the highest levels of anisotropy. The above result about the waves at "intermediate" angles is visually confirmed by Fig. 9, which shows the kinetic energy at large and small scales in the $\theta-\omega$ space, defined as

$$
\begin{aligned}
& E_{K}^{<}(\theta, \omega)=\sum_{k=1}^{8} E_{K}(k, \theta, \omega), \\
& E_{K}^{>}(\theta, \omega)=\sum_{k=9}^{k_{\max }} E_{K}(k, \theta, \omega) .
\end{aligned}
$$

The equivalent quantities for the potential energy, $E_{P}^{<}(\theta, \omega)$ and $E_{P}^{>}(\theta, \omega)$, are also given in Fig. 9. It is evident from Fig. 9 that practically all energy that follows the dispersion relation in the interval $-\theta_{f \text {, } \min }<\theta<\theta_{f \text {,min }}$ is contained in wave numbers between $k=1$ and $k=8$, i.e., in wave numbers smaller than the forcing wave numbers. At the same time, the kinetic energy at large scales, $E_{K}^{<}(\theta, \omega)$, shows a signal centered at $\omega \approx 0$, which, by comparing it to $E_{v}(\theta, \omega)$ in Fig. 8, can be associated to the vortex component of the flow. Since the vortex component is purely horizontal, it does not create density perturbations and so there is no signal at $\omega \approx 0$ in the potential energy $E_{P}^{<}(\theta, \omega)$. For all larger wave numbers $(k>8)$, the energy tends to accumulate in the regions $\theta_{f \text {, min }}<|\theta|<\pi / 2$, which means that, beyond the forcing scales, the vast majority of the energy is concentrated in layered structures.

In summary, consideration of $E_{K}^{<}(\theta, \omega)$ and $E_{P}^{<}(\theta, \omega)$ has shown that the wave signature is predominantly contained in the largest scales of the simulation, in particular at scales larger than the forcing scales. This property of the waves within stratified turbulence in the vicinity of the SST 


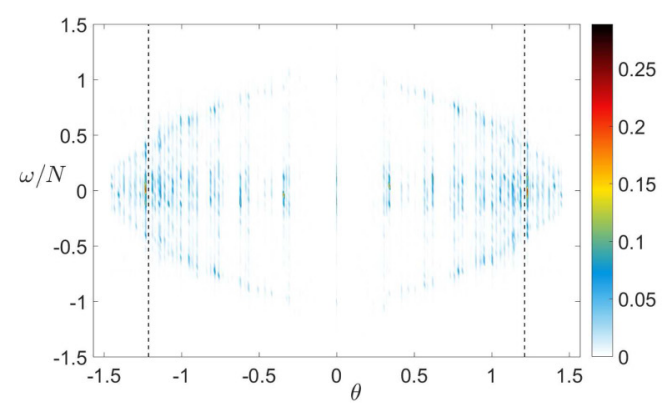

(a)

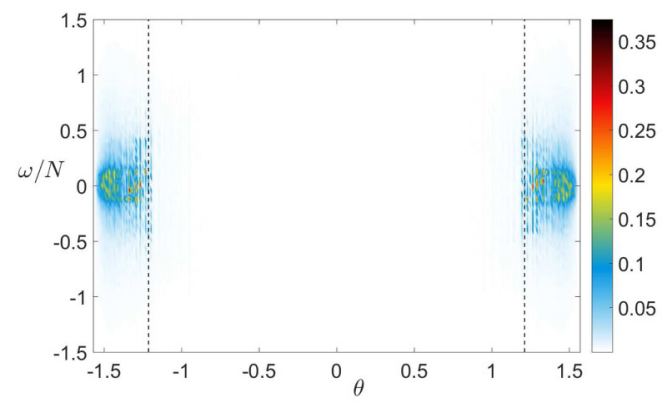

(c)

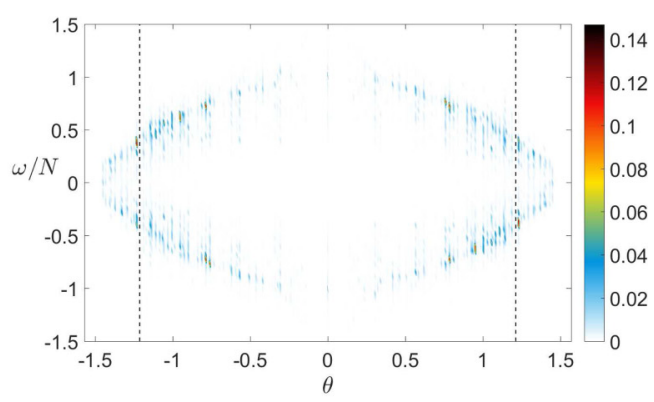

(b)

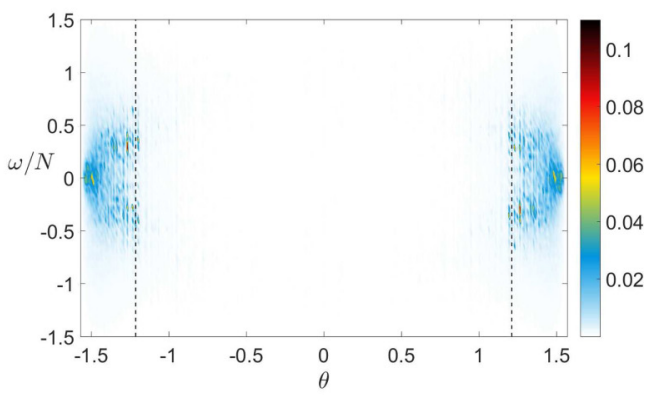

(d)

FIG. 9. Kinetic and potential energy at large and small scales in $\theta-\omega$ space for run R9.9: (a) $E_{K}^{<}(\theta, \omega)$, (b) $E_{P}^{<}(\theta, \omega)$, (c) $E_{K}^{>}(\theta, \omega)$, (d) $E_{P}^{>}(\theta, \omega)$. The vertical dashed lines show the minimum forcing angle, $\theta= \pm \theta_{f, \min }$.

regime is in line with the observation of Lindborg and Brethouwer [14] that waves were mainly present at large scales in their simulations. At the forcing scales and at smaller scales, the energy tends to be concentrated in the regions at "high" angles beyond the forcing angles and at relatively low frequency: This is the signature of the highly anisotropic structures of SST. Note that this result does not imply that there are no waves for $k \geqslant 9$, since plots of $E_{K}(k, \theta, \omega)$ and $E_{P}(k, \theta, \omega)$ in logarithmic scale (not shown) confirm that signatures of the dispersion relation over all $\theta$ continue well beyond the forcing wave numbers. However, when focusing on the region $|\theta|<\theta_{f \text {,min }}$, the clearest peaks and the majority of the energy along the dispersion relation is contained in the first eight wave numbers.

\section{Energy spectra evolution and energy transfers across scales}

We now consider the evolution of the energy spectra over the course of a run and search for an explanation for the large-scale wave signature in terms of energy transfers across scales. We begin by defining the $1 \mathrm{D}$ energy spectra in the horizontal and vertical directions:

$$
\begin{aligned}
& E_{x}\left(\kappa_{x}\right)=\sum_{\substack{k_{y}, k_{z} \\
k_{x}= \pm \kappa_{x}}} \frac{1}{2}\left(\hat{u} \hat{u}^{*}+\hat{v} \hat{v}^{*}\right), \\
& E_{y}\left(\kappa_{y}\right)=\sum_{\substack{k_{x}, k_{z} \\
k_{y}= \pm \kappa_{y}}} \frac{1}{2}\left(\hat{u} \hat{u}^{*}+\hat{v} \hat{v}^{*}\right),
\end{aligned}
$$




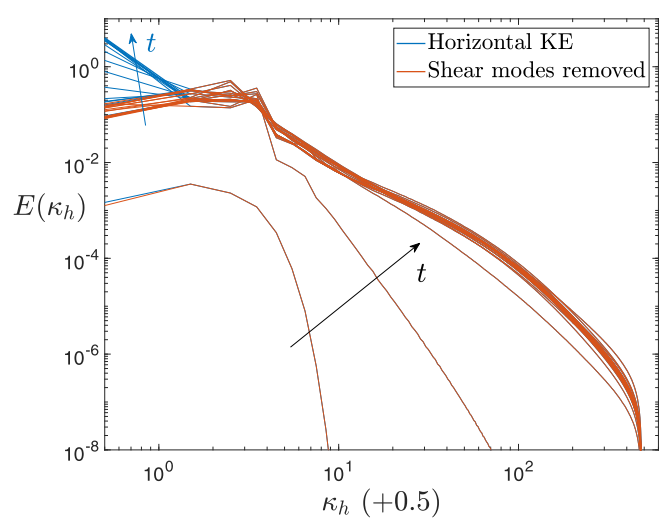

(a)

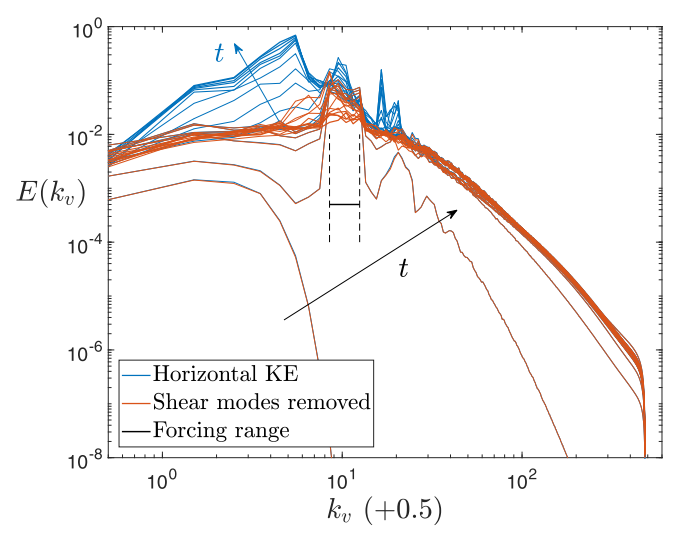

(b)

FIG. 10. Evolution over the course of simulation R3.8 of 1D horizontal and vertical energy spectra: (a) horizontal spectra $E\left(\kappa_{h}\right)$ and (b) vertical spectra $E\left(k_{v}\right)$. In both panels, the energy spectra obtained after filtering out the shear modes are also given. The time at which the spectra are taken is indicated by the line weight of the curves, with increasing line weight corresponding to increasing time. The first spectrum plotted in panels (a) and (b) corresponds to the initial conditions at $t=0$. In both plots, the wave number is shifted by half a wave-number increment to show the spectra at $\kappa_{h}=0$ and $k_{v}=0$. In panel (b), the forcing range in terms of $k_{v}$ is given, while in panel (a) the forcing at $k_{h}=3$ corresponds to the forcing being active from $\kappa_{h}=0$ to $\kappa_{h}=3$.

$$
E\left(k_{v}\right)=\sum_{\substack{k_{x}, k_{y} \\ k_{z}= \pm k_{v}}} \frac{1}{2}\left(\hat{u} \hat{u}^{*}+\hat{v} \hat{v}^{*}\right),
$$

which are spectra of the horizontal kinetic energy $(1 / 2)\left(u^{2}+v^{2}\right)$. A $1 \mathrm{D}$ horizontal spectrum is then defined as $E\left(\kappa_{h}\right)=(1 / 2)\left[E_{x}\left(\kappa_{h}\right)+E_{y}\left(\kappa_{h}\right)\right]$, where $\kappa_{h}$ is a 1D horizontal wave number, not to be confused with the usual (2D) horizontal wave number $k_{h}$. The time evolution of $E\left(\kappa_{h}\right)$ and $E\left(k_{v}\right)$ in run R3.8 is shown in Fig. 10. In this figure, similar horizontal and vertical spectra that do not include the energy in the shear modes $\left(k_{h}=0\right)$ are also given; such spectra with shear modes removed differ only in the first point at $\kappa_{h}=0$ in the case of $E\left(\kappa_{h}\right)$ (by definition) while they differ over a range of vertical wave numbers $k_{v}$ in the case of $E\left(k_{v}\right)$ since the shear modes have a vertical structure. Both energy spectrum evolutions in Fig. 10 highlight that kinetic energy is created via the forcing which, starting from low-level energy spectra at $t=0$ concentrated at low wave numbers, leads to a final state in which a higher energy level is present at all scales. It is also apparent that a stationary state is reached at small scales (high $\kappa_{h}$ and $k_{v}$ ) while there appears to be an energy accumulation at large scales. Such energy accumulation is particularly visible in the continuous growth of $E\left(\kappa_{h}\right)$ at $\kappa_{h}=0$ and of $E\left(k_{v}\right)$ around $k_{v}=5$. A comparison of the evolution of the energy spectra with and without shear modes makes it relatively clear that this accumulation of energy corresponds to the continuous growth of the shear modes. Concerning the growth of the energy spectra over time, it appears that the growth at $\kappa_{h}=0$ and $k_{v}=5$ tends to slow down toward the end of the run. This is reflected by the growth of the kinetic energy of the shear modes, visible in Fig. 6(a), tending to slow down at late times, yet without coming to a halt and reaching stationarity. This continuous growth of energy in the shear modes has been observed by many previous studies involving simulations of stratified turbulence $[9,36,44,46]$. The evidence from previous work and presented herein points toward the growth of energy in the shear modes being due to an upscale energy transfer from the forcing scales. The present work shows that this is both an upscale transfer in the horizontal direction as well as in the vertical direction. Indeed, in the vertical direction, the forcing is active over the range 
$k_{v}=\left[\begin{array}{ll}8 & 12\end{array}\right]$, while $E\left(k_{v}\right)$ is seen to accumulate energy around $k_{v}=5$, with the peak at this wave number becoming significantly higher than the peak over the forcing wave numbers. In physical space, an upscale transfer in the vertical direction for run R3.8 is shown by the fact that the shear modes [see Fig. 4(a)] have a larger vertical scale compared to the forcing (see Fig. 3). The transfer in the horizontal direction is, of course, toward the largest scales of the simulation, $k_{h}=0$, where the domain size limits any further transfers. On the other hand, the transfer in the vertical direction appears to stop before reaching the height of the box at $k_{v}=0$, being concentrated mainly over the wave numbers from $k_{v}=1$ up to the forcing wave numbers.

Turning now to the energy content in the waves, it is informative to consider the energy spectra with shear modes removed, given in Fig. 10, in more detail. We can see that there is not an energy accumulation in such spectra with both horizontal and vertical energy spectra reaching stationarity at some point in time, as expected given that the full kinetic energy with shear modes removed, $E_{K}^{\prime}$, reaches a steady state [see Fig. 6(a)]. At the same time, a relatively high level of energy is present over all horizontal and vertical wave numbers up to the forcing wave numbers and this energy has been created from a much lower energy level at $t=0$. This seems to point toward an energy transfer from the forcing scales to larger scales being active and leading to the $\omega \sim N$ waves. To further this point, we have inspected the kinetic energy in the waves at the largest scales through consideration of $E_{K}(k, \theta, \omega)$, from $k=1$ to $k=8$. The results, shown in Appendix C, appear to confirm that peaks of relatively constant intensity are present along the dispersion relation over all wave numbers $k \in\left[\begin{array}{ll}1 & 8\end{array}\right]$. This justifies a potential interpretation of them being fed by an upscale energy transfer from the forcing scales. This energy transfer may, unlike the transfer to the shear modes, be a transitory event, being active at the beginning of the simulations and then arresting itself as the wave energy reaches stationarity. Conversely, it may also be possible that the upscale energy transfer to the waves is continuous over time, a scenario that is plausible should the large-scale waves participate in the upscale transfer to the shear modes. In this scenario, the waves are continuously excited by an upscale energy transfer and, in turn, transfer their energy in a continuous fashion to the shear modes, where it is ultimately accumulated. Indeed, a recent numerical study of stratified turbulence forced by injecting energy directly in wave motions [47] has proposed, based on the results of their simulations, that energy is transferred to the shear modes via a wave-mean flow interaction. This energy-transfer mechanism would be consistent with a continuous upscale transfer to waves and shear modes in the present DNS.

At this stage, it is important to put such a tentative picture of an upscale energy transfer in stratified turbulence into some perspective: The spectral energy flux quantifies the rate of energy transfer in a certain direction and there is evidence from previous DNS [36,44] that the energy flux to small horizontal scales (downscale) is much larger than the energy flux to large horizontal scales (upscale). Hence, in forced stratified turbulence most of the energy is transferred to small scales and dissipated and only a small percentage of the energy is transferred to large scales, leading to a slow growth of the shear modes and, potentially, a slow transfer to the associated large-scale waves. A detailed analysis of the energy transfers in the simulations, including computation of the spectral energy flux, is beyond the scope of this study. In addition to this energy flux, future work could also attempt to estimate energy exchange rates among three sets of dynamics: shear modes [i.e., $\left.\mathbf{u}_{s m}(z)\right]$, large-scale wave and vortex modes (at scales larger than the forcing), and the remaining part of the velocity field. Attempts at quantifying these exchange rates would, if successful, lead to a better understanding of the mechanism by which shear modes arise in stratified turbulence.

\section{Doppler effect}

From Figs. 9(a) and 9(b), it is clear that for a given $\theta$ the wave signature is distributed over a frequency interval around the dispersion relation frequency. This may be the sign of a Doppler shift of the wave frequency by an advective motion. The Doppler shift of internal gravity waves can be straightforwardly quantified in case of a uniform horizontal advection velocity $\mathbf{U}$, i.e., a uniform horizontal mean flow. The observed frequency is then $\omega=\omega_{D R}+\mathbf{U} \cdot \mathbf{k}$, where $\mathbf{k}$ is the wave vector 


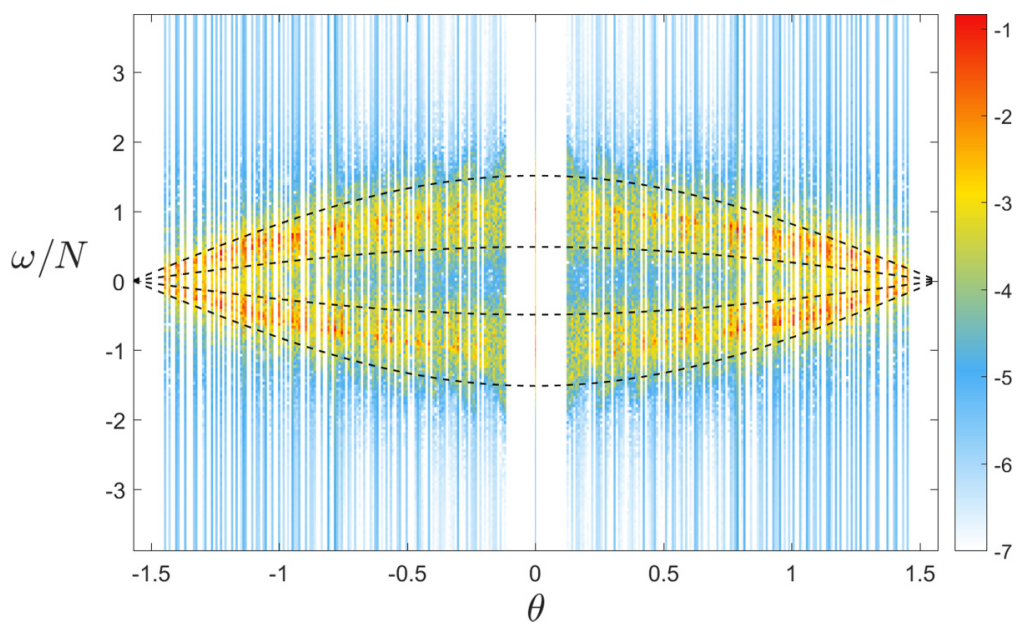

FIG. 11. Color map of $\log _{10}\left(E_{P}^{<}(\theta, \omega)\right)$ for run R9.9 together with plots of $\omega_{\text {Doppler }} / N$ (black dashed lines). The vertical bands in the plot are due to the fact that over this limited number of wave numbers several values of $\theta$ are not sampled. For clarity, values below $10^{-7}$ are filtered out.

of the wave that is advected by the mean flow. Now, in the case of the present DNS, there is a strong horizontal mean flow, which varies vertically and evolves slowly over time, due to the shear modes. This mean flow is not uniform but to a first approximation we can consider that locally the waves see a uniform horizontal flow with magnitude given by $u_{h, s m}=\sqrt{(1 / 2)\left\langle\left|\mathbf{u}_{s m}\right|^{2}\right\rangle}$ (a time average of $\left\langle\left|\mathbf{u}_{s m}\right|^{2}\right\rangle$ within this expression is implied). A similar approach was successfully used when estimating the Doppler shift on the frequency of inertial waves due to large-scale columnar structures in a rotating turbulence experiment [48]. The Doppler-shifted frequency of the waves is then

$$
\omega \approx \omega_{D R}+u_{h, s m} k \cos \theta \cos \alpha,
$$

where $\alpha$ is the (unknown) azimuthal angle of the horizontal projection of $\mathbf{k}$ with respect to the mean flow direction. We are interested in estimating the maximum Doppler shift on waves which are mainly at the smallest wave numbers in the DNS up to $k=8$. We can therefore set $k=8$ in the above expression, as well as $\cos \alpha= \pm 1$, since this will maximize the frequency shift. Thus, the estimated maximum frequency excursions due to the sweeping of the waves by the shear modes are

$$
\omega_{\text {Doppler }}=\omega_{D R} \pm 8 u_{h, s m} \cos \theta= \pm\left(N \pm 8 u_{h, s m}\right) \cos \theta .
$$

We can see that the Doppler shift introduces an amplitude modulation of the $\cos \theta$ term in $\omega_{D R}$, increasing its amplitude above $N$ or decreasing it below $N$. This estimate of the maximum Doppler shift is superimposed on the color map of $E_{P}^{<}(\theta, \omega)$ in Fig. 11, now using a logarithmic scale to show also the wave signatures at smaller orders of magnitude. From this figure, we can appreciate that our estimate for the Doppler shift provides an accurate representation of the behavior of the waves in the DNS run. Overall, the shear modes in the simulation cause a significant Doppler shift and consequent broadening of the dispersion relation.

\section{Wave energy variation with $\mathrm{Re}_{b}$}

The above estimate of the Doppler shift can be used to quantify the energy in the large-scale waves. Limiting our scope to the angles of smaller magnitude than the forcing angles, $-\theta_{f, \min }<$ $\theta<\theta_{f, \min }$, and so to waves with $\omega \sim N$, we can define envelopes in $\theta-\omega$ space within which such waves reside. The two branches of the dispersion relation define an upper wave envelope at positive frequencies and a lower wave envelope at negative frequencies, which of course correspond to a 


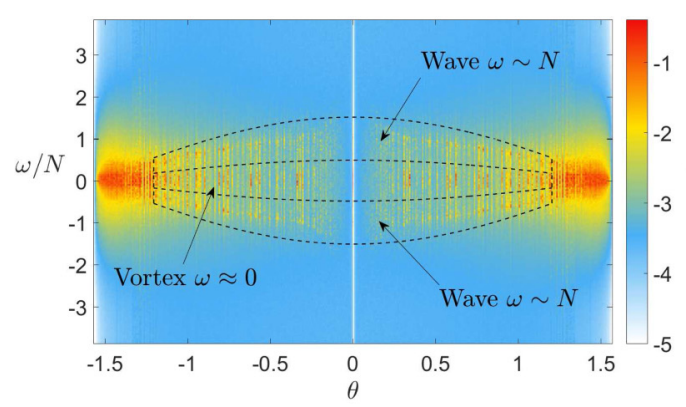

(a)

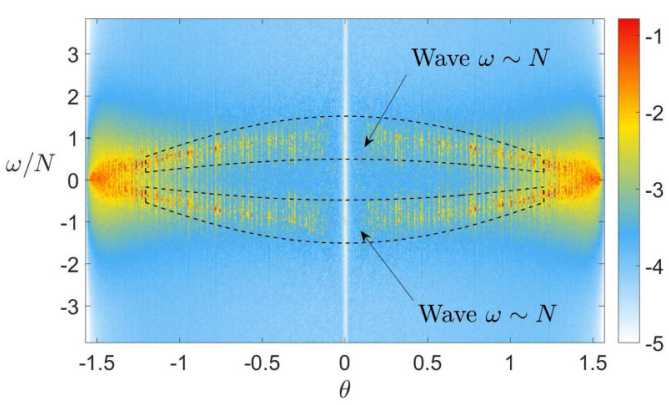

(b)

FIG. 12. Wave and vortex envelopes. The color maps are of data from run R9.9: (a) $\log _{10}\left(E_{K}^{\prime}(\theta, \omega)\right)$ and (b) $\log _{10}\left(E_{P}^{\prime}(\theta, \omega)\right)$. For clarity, values below $10^{-5}$ are filtered out in both color maps.

different sign of $\omega_{\text {Doppler }}$ [see Eq. (9)]. These wave envelopes are depicted in Fig. 12 on top of $E_{K}^{\prime}(\theta, \omega)$ and $E_{P}^{\prime}(\theta, \omega)$ from run R9.9, shown in logarithmic scale. Similarly to the definition of $E_{P}^{\prime}(\theta, \omega), E_{K}^{\prime}(\theta, \omega)=\sum_{k} E_{K}(k, \theta, \omega)$. Over the range of angles $-\theta_{f, \min }<\theta<\theta_{f, \min }$, where there is a relatively large separation between $\omega_{D R}$ and $\omega \approx 0$, the kinetic energy maps in Fig. 7 suggest that there are two distinct dynamical regions: a region corresponding to waves and a region around $\omega \approx 0$ corresponding to the vortex component. We can therefore also define a vortex envelope, located around $\omega=0$ and extending up to the edges of the wave envelopes, as shown in Fig. 12(a). Since the vortex component is expected to possess kinetic energy but no potential energy, we define the vortex envelope only for the kinetic energy maps.

With the above definitions of the wave envelopes, we can now define an estimate for the kinetic energy in the large-scale $\omega \sim N$ waves:

$$
E_{K, \omega \sim N}=\sum_{-\theta_{f, \text { min }}}^{\theta_{f, \min }} \sum_{\left(N-8 u_{h, s m}\right) \cos \theta}^{\left(N+8 u_{h, s m}\right) \cos \theta} E_{K}(\theta, \omega)+\sum_{-\theta_{f, \text { min }}}^{\theta_{f, \text { min }}} \sum_{-\left(N+8 u_{h, s m}\right) \cos \theta}^{-\left(N-8 u_{h, s m}\right) \cos \theta} E_{K}(\theta, \omega) .
$$

The first term is the sum over the upper wave envelope and the second term is the sum over the lower wave envelope, and for each term the inner sum is over $\omega$ and the outer sum is over $\theta$. We define the potential energy in the $\omega \sim N$ waves, $E_{P, \omega \sim N}$, in an analogous fashion, starting from $E_{P}(\theta, \omega)$. Similarly, we can define the vortex kinetic energy, $E_{K, \omega \approx 0}$, as the sum of $E_{K}(\theta, \omega)$ over the vortex envelope:

$$
E_{K, \omega \approx 0}=\sum_{-\theta_{f, \min }}^{\theta_{f, \min }} \sum_{-\left(N-8 u_{h, s m}\right) \cos \theta}^{\left(N-8 u_{h, s m}\right) \cos \theta} E_{K}(\theta, \omega)
$$

Note that the definition of the wave and vortex envelopes is consistent with the energy concentration of the wave and vortex components shown in Fig. 8, so that we could equally have used the wave envelope on the wave component kinetic energy map, $E_{w}(\theta, \omega)$, to define $E_{K, \omega \sim N}$ or the vortex envelope on $E_{v}(\theta, \omega)$ to define $E_{K, \omega \approx 0}$. This is the result of the fact that, for angles $-\theta_{f, \min }<\theta<\theta_{f, \min }$, the wave-vortex decomposition is valid and gives a reliable description of the dynamics.

Assuming that all three DNS runs are similarly characterized by waves at scales larger than the forcing scales that are swept by the shear modes, we apply the above decomposition of $E_{K}(\theta, \omega)$ and $E_{P}(\theta, \omega)$ to all runs. As a test of the relevance of this decomposition also to the lower $\operatorname{Re}_{b}$ runs, we show the wave and vortex envelopes for run R1.6 in Fig. 13. This figure shows that the energy around the dispersion relation is indeed confined to the wave envelopes and that the energy at $\omega \approx 0$ is contained within the vortex envelope. We hence calculate $E_{K, \omega \sim N}, E_{P, \omega \sim N}$, and $E_{K, \omega \approx 0}$ for the 


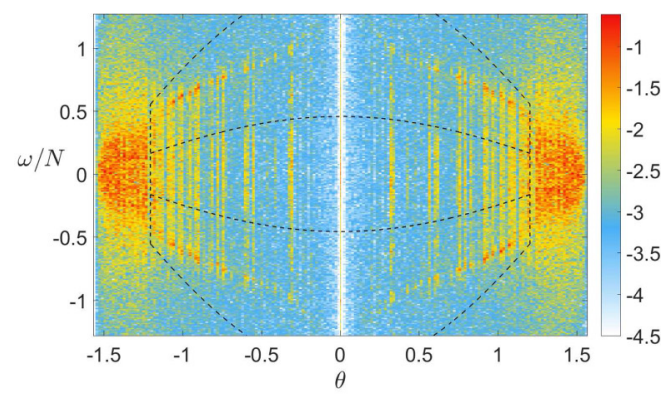

(a)

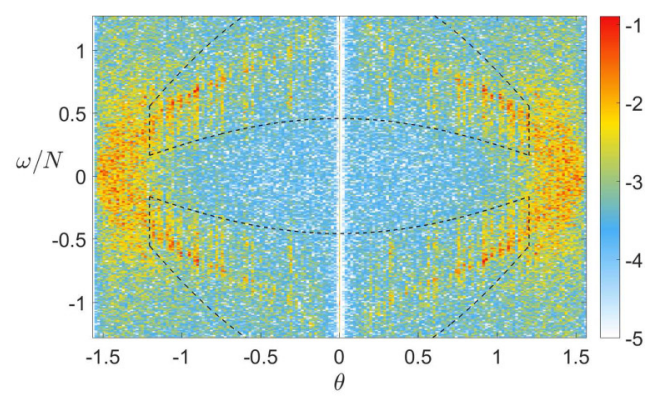

(b)

FIG. 13. Wave and vortex envelopes for run R1.6 superimposed on color maps of (a) $\log _{10}\left(E_{K}(\theta, \omega)\right)$ and (b) $\log _{10}\left(E_{P}(\theta, \omega)\right)$. For clarity, values below $10^{-4.5}$ in panel (a) and below $10^{-5}$ in panel (b) are filtered out.

three DNS runs. This allows us to check what is the effect of increasing $\operatorname{Re}_{b}$ on the energy of the $\omega \sim N$ waves in the simulation. The evolution of the wave energy and of the vortex kinetic energy as a function of $\operatorname{Re}_{b}$ is given in Fig. 14(a). All quantities are normalized by the overall kinetic energy with shear modes removed, $E_{K}^{\prime}$. It is clear that the energy in the $\omega \sim N$ waves decreases with increasing $\mathrm{Re}_{b}$; this behavior is true both for kinetic and potential energy, and the greatest decrease is seen in going from run $\mathrm{R} 3.8$ to $\mathrm{R} 9.9$, reaching $\mathrm{Re}_{b} \approx 10$. The fraction of kinetic energy in the waves with respect to $E_{K}^{\prime}$ drops from about $23 \%$ in run $\mathrm{R} 1.8$ to about $14 \%$ in run $\mathrm{R} 9.9$. This confirms the general wisdom that waves become less important as we enter the SST regime, as the energy is more concentrated in the highly anisotropic layers. This statement, of course, concerns the high-frequency waves with $\omega \sim N$ only as we are not able to draw clear conclusions about the low-frequency waves, which are difficult to disentangle from the vortices in an unambiguous way. Equipartition of wave kinetic and potential energy is not observed, with the kinetic energy being systematically higher than the potential energy. This result may in part be due to the fact that $E_{K, \omega \sim N}$ and $E_{P, \omega \sim N}$ contain energy from all wave numbers, not only from the largest scales. This means that also energy at small scales with $k>k_{o}$, which cannot be associated to waves, is included

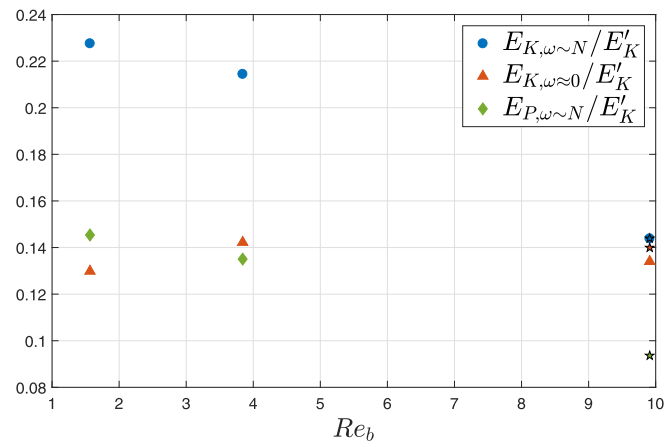

(a)

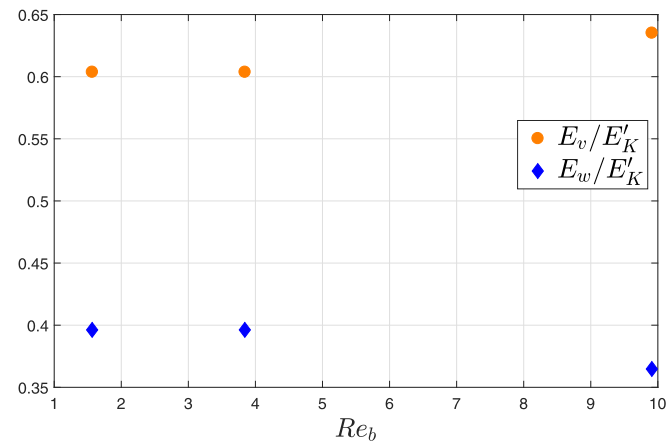

(b)

FIG. 14. Wave and vortex energy across the three DNS runs shown as a function of $\operatorname{Re}_{b}$. (a) Kinetic and potential energy of $\omega \sim N$ waves and kinetic energy of $\omega \approx 0$ vortices. The star symbols at $\operatorname{Re}_{b}=9.9$ indicate the wave and vortex kinetic energy calculated from $E_{K}^{\prime}(\theta, \omega)$ instead of $E_{K}(\theta, \omega)$, and the wave potential energy calculated from $E_{P}^{\prime}(\theta, \omega)$ instead of $E_{P}(\theta, \omega)$. The color code for these quantities is maintained. (b) Wave and vortex kinetic energy $E_{w}$ and $E_{v}$ as obtained from the wave-vortex decomposition. In both panels, the energy is normalized by the overall kinetic energy not counting the shear modes, $E_{K}^{\prime}$. 
in the wave kinetic and potential energy (here $k_{o}=\sqrt{N^{3} / \epsilon}$ is the Ozmidov wave number, beyond which buoyancy effects are negligible). This may create a bias in the numeric values of $E_{K, \omega \sim N}$ and $E_{P, \omega \sim N}$. For what concerns the vortex kinetic energy, $E_{K, \omega \approx 0}$, this quantity remains approximately constant as we increase $\operatorname{Re}_{b}$. The waves have more kinetic energy than the vortices at $\operatorname{Re}_{b} \sim 1$ but, as $\mathrm{Re}_{b}$ increases and the wave energy decreases, approximate equipartition between wave and vortex kinetic energy is reached at $\operatorname{Re}_{b} \approx 10$. This final result may be coincidental and should be further explored.

The tentative picture that emerges is that the small "leak" of energy, from the anisotropic forcing scales to larger scales with smaller $|\theta|$, is decreased as the buoyancy Reynolds number is increased and we enter the SST regime. Nonetheless, even at $\operatorname{Re}_{b} \approx 10$ the energy in the $\omega \sim N$ waves fed by this small upscale energy transfer is non-negligible. This is an interesting feature of the present simulations that was not expected according to the SST theory, which predicts anisotropic vortices and waves fed by a purely downscale energy transfer [11,12].

Finally, we consider the wave and vortex kinetic energies, $E_{w}$ and $E_{v}$, as obtained from the wave-vortex decomposition, across the three runs at different $\operatorname{Re}_{b}$. We compute time averages of $E_{w}$ and $E_{v}$ over the steady-state period [a typical time evolution of $E_{w}$ and $E_{v}$ is shown in Fig. 6(b)]. These quantities are plotted in Fig. 14(b). The first point to note when comparing $E_{w}$ and $E_{v}$ to the equivalent quantities in Fig. 14(a) is that the energy levels of $E_{w}$ and $E_{v}$ are much higher. This is, of course, due to the fact that to obtain them we sum the energy in $\hat{\mathbf{u}}_{w}(\mathbf{k})$ and $\hat{\mathbf{u}}_{v}(\mathbf{k})$ over all $\theta$ and therefore include the highly energetic regions in the range $\theta_{f \text {, min }}<|\theta|<\pi / 2$. Furthermore, it can be seen that $E_{w}$ and $E_{v}$ do not evolve significantly with $\operatorname{Re}_{b}$ and that the prevalence of $E_{v}$ over $E_{w}$ is maintained over the entire range of $\mathrm{Re}_{b}$. There appears to be a small increase of the fraction of $E_{v}$ with respect to $E_{K}^{\prime}$ as we reach $\mathrm{Re}_{b} \approx 10$, with an associated small decrease of the $E_{w}$ fraction (recall that $E_{K}^{\prime}=E_{w}+E_{v}$ ). However, these variations are significantly smaller than the variations of $E_{K, \omega \sim N}$ and $E_{P, \omega \sim N}$ in Fig. 14(a), both in relative and absolute terms. This different behavior is due to the energy in the region $\theta_{f, \min }<|\theta|<\pi / 2$, which dominates $E_{w}$ and $E_{v}$, but is purposely excluded from $E_{K, \omega \sim N}, E_{P, \omega \sim N}$, and $E_{K, \omega \approx 0}$. The fact that all wave vectors $\mathbf{k}$ and thus all angles $\theta$ are summed over to obtain $E_{w}, E_{v}$ can represent a limitation of global energy budgets obtained directly from the wave-vortex decomposition. In the present case, this means that the evolution of the wave energy $E_{K, \omega \sim N}$ and $E_{P, \omega \sim N}$ shown in Fig. 14(a) is not clearly reproduced in Fig. 14(b), while it is believed to be a true physical feature of the current DNS. Of course, this limitation is of particular importance in the SST regime, where most of the energy tends to accumulate in the specific region of the $(\theta, \omega)$ plane close to $\theta= \pm \pi / 2$ (and $\omega \approx 0$ ).

\section{Preferred directions of wave propagation}

It is relatively clear from Figs. 7-9 that the wave energy has distinct peaks, corresponding to specific wave-number orientations $\theta$. This implies that the waves have a number of preferred directions of propagation, that is to say, a number of directions of propagation at which they are preferentially excited within homogeneous stratified turbulence. To take a closer look at this, we have calculated the maxima of $E_{K}(\theta, \omega)$ and $E_{P}(\theta, \omega)$ for every $\theta$, limiting our range of frequencies to be within the wave envelopes (because of the symmetry we consider only the top envelope). We have hence computed the quantities $E_{P, \max }(\theta)$ and $E_{K, \max }(\theta)$, which are plotted in Figs. 15 and 16, respectively. For run $\mathrm{R} 9.9$, we have computed the quantities $E_{P, \text { max }}^{\prime}(\theta)$ and $E_{K, \text { max }}^{\prime}(\theta)$, as the maxima of $E_{P}^{\prime}(\theta, \omega)$ and $E_{K}^{\prime}(\theta, \omega)$ within the wave envelope.

Focusing first on the potential energy, which should be less influenced by the forcing at angles close to $\theta_{f \text {,min }}$, we can see that the energy peaks at a series of angles that recur across the three simulations. In addition to a peak at $\theta=0^{\circ}$, corresponding to zero-group-velocity waves with $\omega=N$, peaks are frequently observed at $\theta= \pm 45^{\circ}$ and within the range $\pm\left[52^{\circ} 54.5^{\circ}\right]$. Similar observations can be made when turning to the kinetic energy maxima, even though there are also pronounced peaks close to the boundaries of the wave envelope, $\theta= \pm \theta_{f \text {, min }}$, which are thought to be associated with the forcing. It is important to realize that the second group of waves, with 


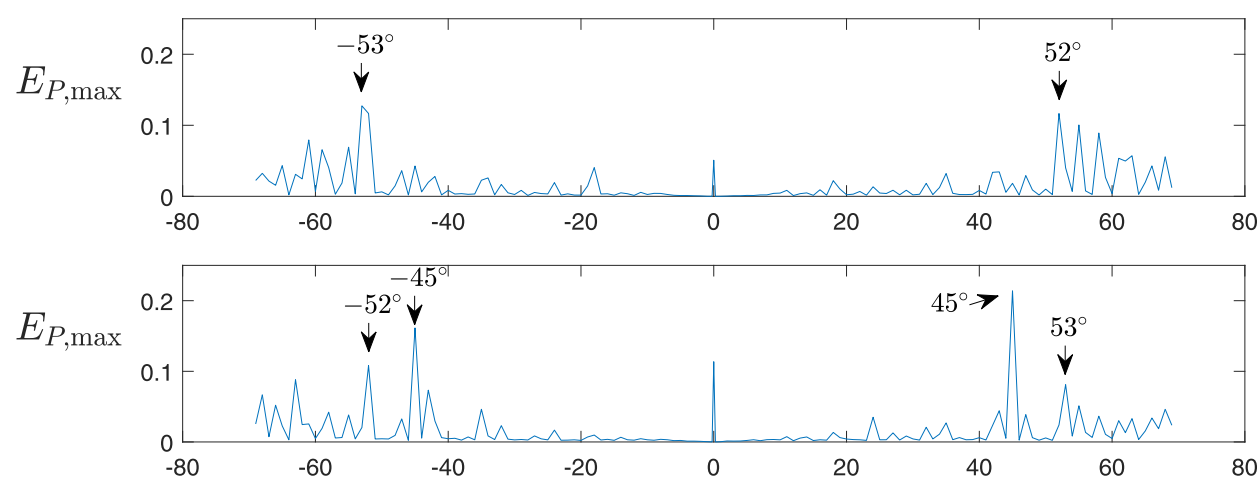

R1.6

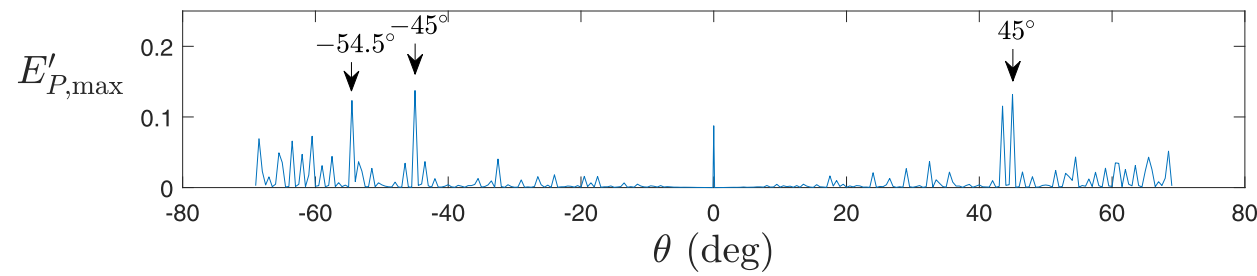

R9.9

FIG. 15. Plots of $E_{P, \max }(\theta)$ and of $E_{P, \max }^{\prime}(\theta)$ (in case of run R9.9), the maxima of the potential energy within the wave envelope as a function of $\theta$. The corresponding run is indicated on the right of each plot.

$\theta= \pm\left[52^{\circ} 54.5^{\circ}\right]$, transport energy according to their group velocity $\mathbf{c}_{g}$, which is oriented at an angle $\phi=90^{\circ}-\theta= \pm\left[35.5^{\circ} 38^{\circ}\right]$ with respect to the horizontal. This means that these waves actually transport energy in "close-to-horizontal" directions, a somewhat intuitive result for a strongly stratified flow. Another remark that can be made when considering the plots in Figs. 15 and 16 is that the wave energy peaks become more distinct when moving to higher $\operatorname{Re}_{b}$, i.e., toward
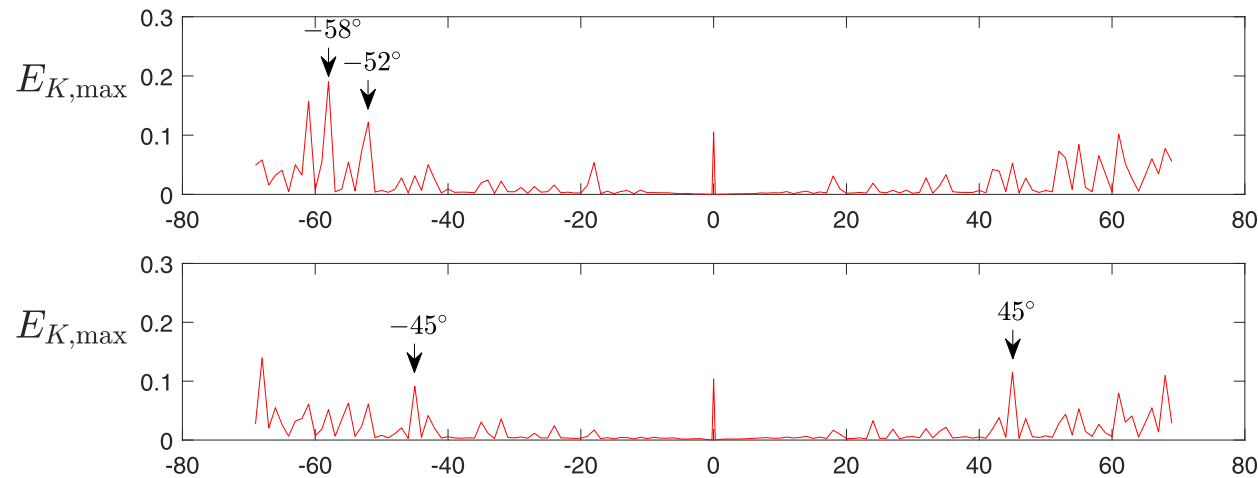

$\mathrm{R} 3.8$

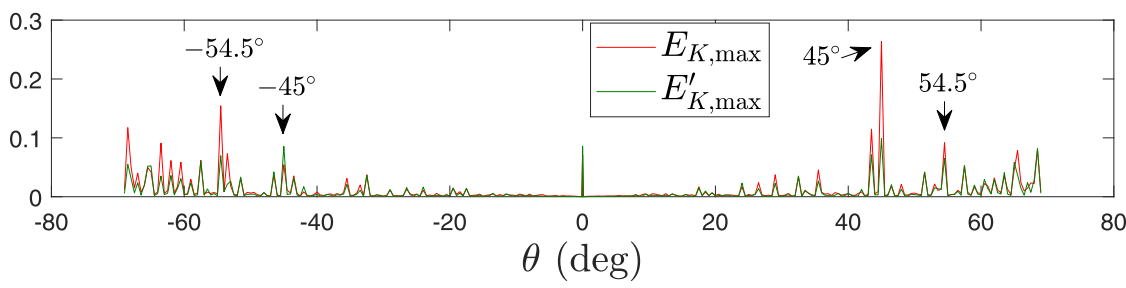

R9.9

FIG. 16. Plots of $E_{K, \max }(\theta)$ and of $E_{K, \max }^{\prime}(\theta)$ (in case of run R9.9). 
a more vigorously turbulent state. Note also that, in the case of run R9.9, the same peaks presented by $E_{K, \max }(\theta)$ are shown by $E_{K, \max }^{\prime}(\theta)$, which should be a more accurate measure, but the intensity of the peaks is decreased in $E_{K, \max }^{\prime}(\theta)$, typically.

Very similar propagation directions have been observed in the problem of internal gravity wave radiation from a localized region of turbulence. In experiments with grid-generated turbulence, waves were seen to propagate in the linearly stratified region below a turbulent mixed region, with propagation angles in the range $\theta=42-55^{\circ}$ [27]. In the case of a turbulent wake in a stratified fluid, experiments showed waves propagating at an angle $\theta \approx 55^{\circ}$ [29]. In a numerical study of the stratified turbulent wake [30], a larger range of propagation angles $\theta$ was observed, with $\theta$ depending both on time and on the wake Reynolds number; the overall range of observed angles was $\theta=\left[26^{\circ} 50^{\circ}\right]$. A further relevant work is a primarily numerical study of a confined region of stratified turbulence, for which the propagation angles were in the range $\theta=50-55^{\circ}$ [28]. The presence of similar wave directions in our DNS of homogeneous stratified turbulence is an interesting observation. It is suggestive of the fact that these waves are generated in a large class of problems in turbulence in a stratified fluid. This observation suggests that in the case where the localized region of turbulence is itself stably stratified [28-30], it is likely that the waves are generated in the quasihomogeneous interior of the turbulence and then subsequently "exit" the turbulent region continuing to propagate in the ambient stratified fluid. The picture is more complex in the case of the turbulence being generated in a constant density fluid above a quiescent stratified fluid [27], since the turbulence interior is not wave-bearing but the entrainment of stratified fluid into the turbulent region may be important.

Possible explanations for the specific propagation directions of the waves have been reported. Reference [29] attributes the observed propagation angle of $\theta \approx 55^{\circ}$ to the fact that the wave amplitude $A_{\Delta z}$ in terms of isopycnal displacement is maximized for an impulsive point source at $\theta=\tan ^{-1} \sqrt{1 / 3} \approx 54.7^{\circ}$. On the other hand, it was shown by Dohan and Sutherland [27] that $\theta=45^{\circ}$ maximizes the vertical transport of horizontal momentum for a given amplitude $A_{\Delta z}$. In their work, they also suggest that $\theta \approx 35^{\circ}$ maximizes the vertical energy flux [27]. However, regarding this second point, wave propagation angles of $\theta \approx 35^{\circ}$ were not observed in their experiments or in two of the previously mentioned studies [28,29] or in the present DNS. Some evidence for a preferred wave propagation at $\theta \approx 31^{\circ}$ is provided by the numerical wake study but this angle is selected only when the wake is at low Reynolds number [30]. We here focus on the waves at $\theta \approx 55^{\circ}$, observed in previous studies and our current work, and provide an alternative explanation to the impulsive point source argument [29], building on the reasoning of Dohan and Sutherland [27]. Consider a plane internal gravity wave, propagating in the $x$ - $z$ plane. Its horizontal energy flux, $F_{x}=E c_{g, x}$, where $E$ is the wave energy (kinetic plus potential energy) averaged over a period and $c_{g, x}$ is the horizontal component of the group velocity, can be expressed in terms of $A_{\Delta z}$ and $k_{x}$ as (cf. the expression for the vertical energy flux in Dohan and Sutherland [27]):

$$
F_{x}=\frac{1}{2} \frac{A_{\Delta z}^{2} N^{3}}{k_{x}} \sin ^{2} \theta \cos \theta
$$

For a fixed $A_{\Delta z}$ and $k_{x}, F_{x}$ is maximum at $\theta \approx 54.7^{\circ}$. This may explain why, in the case where turbulence creates a vertical displacement and hence generates a wave with amplitude $A_{\Delta z}$, the wave tends to propagate at $\theta \approx 55^{\circ}$. The fact that we are keeping $k_{x}$ constant (instead of $k$ or $k_{z}$ ) is consistent with this wave generation mechanism since it appears reasonable that the horizontal scale of the vertical disturbance will determine the horizontal wavelength of the excited wave so that $k_{x}$ is fixed by the turbulence. Then, the physical rationalization for this wave angle selection is that most of the advection in stratified turbulence occurs in layers, which include the shear modes, that transport momentum and energy horizontally and so waves at $\theta \approx 55^{\circ}$ may be able to extract energy most efficiently from these horizontal motions. This mechanism may involve a resonant feedback between the waves and the turbulence as described by Dohan and Sutherland [27]. 


\section{DISCUSSION AND CONCLUSIONS}

In this work, we have found wave signatures in homogeneous stratified turbulence over the entire frequency range of the dispersion relation of internal gravity waves, from $\omega \approx 0$ to $\omega=N$. We have isolated the set of waves with the clearest signature in the $(\theta, \omega)$ plane as being relatively high-frequency waves with $\omega \sim N$ which reside at scales larger than the forcing scales, i.e., at the largest scales of the numerical domain. This extends previous work [14] in which evidence of the presence of these waves had been found, as we here provide a systematic study of the properties of the $\omega \sim N$ waves through the use of a spatiotemporal analysis taking into account all modes and all $\mathbf{k}$ within the DNS. This detailed analysis has clarified that the waves and their associated low-frequency vortex motions contain a significant part of the overall kinetic energy with shear modes filtered out (up to $\approx 35 \%$ when summing wave and vortex kinetic energy). This wave energy, however, was found to decrease across the simulations as $\operatorname{Re}_{b}$ is increased up to $\operatorname{Re}_{b} \approx 10$ and the SST regime is approached. Moreover, these large-scale waves are preferentially excited at specific propagation angles, namely $\theta=45^{\circ}$ and $\theta=\left[52^{\circ} 54.5^{\circ}\right]$. Interestingly, very similar propagation directions have been observed previously by studies of inhomogeneous stratified turbulence, in which waves generated by a confined turbulent region are radiated in a surrounding stratified environment [27-29]. Such equivalent observations in different types of stratified turbulent flows are suggestive of a generic mechanism existing for internal gravity wave generation by turbulence. However, this equivalence does not seem to carry through exactly to recent numerical studies of stratified turbulent wakes [30,49], including a very recent work [49] in which the wave propagation angles were found to cover a much wider range.

Over the range of $\theta$ between the forcing regions, $-\theta_{f, \min }<\theta<\theta_{f, \text { min }}$ (a range which is sufficiently distinct from $\theta= \pm \pi / 2)$, the wave-vortex decomposition appears to provide an accurate description of the wave $\left(\omega=\omega_{D R}\right)$ and vortex $(\omega \approx 0)$ dynamics, as highlighted by the spatiotemporal analysis of the wave and vortex components, $\mathbf{u}_{w}$ and $\mathbf{u}_{v}$. This finding is in agreement with a similar observation by Lindborg and Brethouwer [14] based on frequency spectra of wave and vortex components from a set of large-scale modes. The reason for this good agreement in a highly nonlinear flow is thought to be that in this region of the $(\theta, \omega)$ plane there is a significant scale separation between wave frequency $(\omega \sim N)$ and vortex frequency $(\omega \approx 0)$. Indeed, at low Froude number, if the vortex timescale is the eddy turnover time $\tau$, this frequency or timescale separation is of the order of $\tau / N^{-1} \sim \mathrm{Fr}_{h}^{-1} \gg 1$. Hence, in SST, if energy is transferred to this region at "intermediate" angles $\theta$, the conditions for the wave-vortex decomposition to be valid will be met. The question is then how is the energy transferred from the relatively high $|\theta|$ of the forcing to smaller $|\theta|$ as well as to larger spatial scales corresponding to the wave signature. In terms of the transfer to larger scales, we have proposed that this occurs through an upscale energy transfer from the forcing scales which excites wave and vortex modes at larger scales. The waves may in turn be responsible for the ultimate growth and accumulation of energy in the shear modes, via a wave-mean flow interaction, as has been proposed by a recent numerical study [47]. The fact that the shear modes grow through an upscale energy transfer has been confirmed through consideration of the horizontal and vertical energy spectra, $E\left(\kappa_{h}\right)$ and $E\left(k_{v}\right)$, and their evolution over time. This transfer has been shown to be upscale in terms of both horizontal and vertical scales, even though the upscale transfer in the vertical direction may be limited to scales close to the forcing scales since it arrests itself at the largest scale of the domain. On the other hand, the horizontal upscale transfer reaches the largest scales of the box $\left(k_{h}=0\right)$, indicating that this transfer would potentially carry on to even larger horizontal scales. The shear modes also have a distinct effect on the large-scale waves since they are found to cause a Doppler shift of their frequency. We have been able to successfully quantify this Doppler shift in $\omega_{D R}$ by considering the shift to be due to a locally uniform horizontal flow with speed given by the rms velocity of the shear modes.

The simulations we have carried out have a buoyancy Reynolds number varying from $\operatorname{Re}_{b} \approx 1.5$ to $\operatorname{Re}_{b} \approx 10$. Some variation of the results has been observed with increasing $\operatorname{Re}_{b}:$ A reduction of the energy in the large-scale waves as quantified through our "wave envelope" approach and 
what appears to be a slightly higher concentration of the energy in the anisotropic motions with $\theta_{f \text {, } \min }<|\theta|<\pi / 2$ (see Fig. 7). As discussed previously, the energy budgets from the wave-vortex decomposition, $E_{w}$ and $E_{v}$, evolve very little with increasing $\mathrm{Re}_{b}$. The results will probably undergo (further) variations as $\mathrm{Re}_{b}$ is increased beyond $\mathrm{Re}_{b}=10$, while maintaining a low Froude number. In particular, some of the trends observed herein may be consolidated and/or some further or contrasting variations may occur. For example, a quantity that we have not investigated in detail, the vertical wave number (or scale) of the shear modes may also depend on $\mathrm{Re}_{b}$, and so variations of this quantity may result from a further increase in $\mathrm{Re}_{b}$. This is something worth examining in future work.

A limitation of the present study is that we have only considered one forcing $\mathbf{f}$, specifically one set of forcing angles $|\theta|=\left[\theta_{f, \min } \theta_{f, \max }\right]$. As can be seen in Fig. 7, the forcing angles represent the boundary between two distinct dynamical regions: one region with $|\theta|<\theta_{f \text {,min }}$ and at scales larger than the forcing $(k<9)$ at which waves and vortices are clearly observed, and a second region within the range $\theta_{f, \min }<|\theta|<\pi / 2$ and at smaller scales $(k \geqslant 9)$ at which anisotropic structures dominate the energetics and wave and vortex signatures are not clearly discernible. Even though these two dynamical regions are thought to be a general feature of stratified turbulence in this parameter range, exploring different forcing angles and scales would allow one to get a more complete picture of this flow and test some of the results obtained in this study. Moreover, the present forcing has equal magnitudes in the wave and vortex components of the flow. A forcing differing in this respect may also affect some of the results, particularly if the forcing is concentrated entirely in one of these two components, a situation which is often considered in the study of SST $[3,14,16,36,38]$. A further limitation is the inevitable finiteness of the period of observation of the waves $T$ in the simulations. In particular, this period $T$ may have implications on our ability to capture the very slow motions at $\omega \sim \tau^{-1} \approx 0$, represented by the vortex modes as well as the motions of the layers of SST. Future work could employ longer time periods for the spatiotemporal analysis in order to provide more definitive results on these slow motions in stratified turbulence. Finally, it is worth highlighting the unsolved problem of disentangling waves from vortices in the highly energetic regions beyond the forcing angles, at $\theta_{f, \min }<|\theta|<\pi / 2$. The present results are not clear cut in terms of isolating the signature of the dispersion relation in this region, even though the dispersion relation has been observed in $E_{P}(\theta, \omega)$ and in the wave-component map $E_{w}(\theta, \omega)$ over these angles. This lack of clarity has led us to refrain from attempting to create wave and vortex energy budgets in this region of the $\theta-\omega$ space. More work is needed to investigate the dynamics of waves and vortices in this region corresponding to the layers of SST and to determine whether it is actually possible to unambiguously isolate the wave dynamics from the vortex dynamics under such highly anisotropic conditions. This open question also leads us to express a recommendation to use caution when interpreting global energy budgets obtained from the wave-vortex decomposition, especially in the SST regime. This is because such budgets sum over all motions, from intermediate to high $\theta$ and from small to large scales, and hence may not provide an accurate representation of the energetics of waves and vortices in a stratified turbulent flow in which more than one dynamical region is present.

\section{ACKNOWLEDGMENTS}

We would like to thank Henri Lam for helping us carry out the 4D analysis of one of the runs. The suggestions of two anonymous referees helped improve this manuscript considerably and are gratefully acknowledged. This work was supported by the LABEX iMUST (ANR-10-LABX-0064) of Université de Lyon, within the program "Investissements d'Avenir" (ANR-11-IDEX-0007) operated by the French National Research Agency (ANR). Computational resources on Joliot-Curie at TGCC, France, were granted by GENCI under a "Grands Challenges" allocation and resources at IDRIS, France, were granted by GENCI under Allocation No. A0042A02206. Further HPC resources were provided at PMCS2I in Lyon, France, and were granted by the Fédération Lyonnaise de Modélisation et Sciences Numériques (FLMSN), partner of the EQUIPEX EQUIP@ MESO. 


\section{APPENDIX A: FORMULATION OF THE WAVE-VORTEX DECOMPOSITION}

Starting from the reduced equations for the wave and vortex components, it can be shown (see Ref. [7]) that the wave component is vertically irrotational (vertical vorticity $\omega_{z}=0$ ) and contains all the horizontal divergence, $\partial u / \partial x+\partial v / \partial y$, of the flow, while the vortex component is horizontally nondivergent and contains all the $\omega_{z}$. Because of this property, the components of the velocity field in Fourier space, projected onto the Craya-Herring reference frame, correspond exactly to the wave and vortex components $\hat{u}_{w}$ and $\hat{u}_{v}$. The Craya-Herring reference frame is a reference frame based on the wave vector $\mathbf{k}$ and the vertical direction; it has unit vectors (see, e.g., Ref. [50])

$$
\begin{gathered}
\mathbf{e}_{1}=\frac{\mathbf{k} \times \mathbf{e}_{z}}{\left|\mathbf{k} \times \mathbf{e}_{z}\right|}=\frac{k_{y}}{k_{h}} \mathbf{e}_{x}-\frac{k_{x}}{k_{h}} \mathbf{e}_{y}, \\
\mathbf{e}_{2}=\frac{\mathbf{k} \times \mathbf{e}_{1}}{\left|\mathbf{k} \times \mathbf{e}_{1}\right|}=\frac{k_{x} k_{z}}{k_{h} k} \mathbf{e}_{x}+\frac{k_{y} k_{z}}{k_{h} k} \mathbf{e}_{y}-\frac{k_{h}}{k} \mathbf{e}_{z}, \\
\mathbf{e}_{3}=\frac{\mathbf{k}}{|\mathbf{k}|}=\frac{k_{x}}{k} \mathbf{e}_{x}+\frac{k_{y}}{k} \mathbf{e}_{y}+\frac{k_{z}}{k} \mathbf{e}_{z} .
\end{gathered}
$$

The incompressibility condition on the velocity field, $\boldsymbol{\nabla} \cdot \mathbf{u}=0$, translates to the condition $\mathbf{k} \cdot \hat{\mathbf{u}}=0$ in Fourier space. This means that $\hat{\mathbf{u}} \cdot \mathbf{e}_{3}=0$ and that $\hat{\mathbf{u}}$ can be written using only two components, in the $\mathbf{e}_{1}$ and $\mathbf{e}_{2}$ directions. Now, it is straightforward to confirm that the component in the $\mathbf{e}_{1}$ direction is horizontally nondivergent and has $\omega_{z} \neq 0$ and that the component in the $\mathbf{e}_{2}$ direction has $\omega_{z}=0$ and nonzero horizontal divergence. Hence, we can write the wave-vortex decomposition as $\hat{\mathbf{u}}=\hat{\mathbf{u}}_{v}+\hat{\mathbf{u}}_{w}=\hat{u}_{v} \mathbf{e}_{1}+\hat{u}_{w} \mathbf{e}_{2}$.

As mentioned previously, it is important to note that modes with $\mathbf{k}=\left[0,0, k_{z}\right]$, which correspond to a horizontal flow that is horizontally invariant but maintains a $z$ dependence, cannot be expressed in the Craya-Herring reference frame. These modes are known as the shear modes $[9,36]$ and we will treat them separately. The expression for the full velocity field in Fourier space thus needs to be revised to $\hat{\mathbf{u}}=\hat{\mathbf{u}}_{v}+\hat{\mathbf{u}}_{w}+\hat{\mathbf{u}}_{s m}$.

\section{APPENDIX B: APPROXIMATION IN REDUCED SPATIOTEMPORAL ANALYSIS}

The kinetic energy obtained from our reduced spatiotemporal analysis, $E_{K}(\theta, \omega)$, is an approximation to the true kinetic energy obtained by reducing the $4 \mathrm{D}$ quantity $E_{K}(\mathbf{k}, \omega)=(1 / 2) \tilde{\mathbf{u}}(\mathbf{k}, \omega)$. $\tilde{\mathbf{u}}(\mathbf{k}, \omega)^{*}$ to the $2 \mathrm{D}$ space given by the $(\theta, \omega)$ coordinates. This is because by performing the sum of velocity Fourier modes over conical shells before taking the square to form an energy, we introduce a number of cross terms, which are the products of different Fourier modes within the same conical shell. Since both summation and Fourier transformation are linear operations, we can consider that

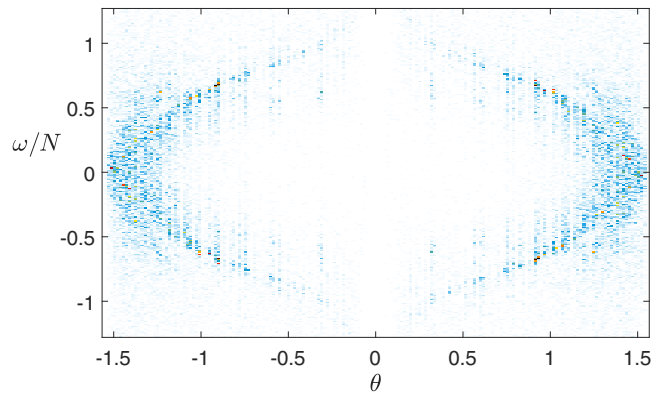

(a)

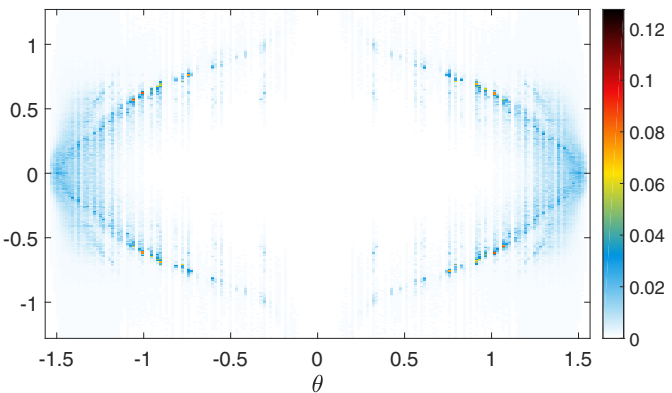

(b)

FIG. 17. Potential energy $E_{P}(\theta, \omega)$ for run R1.6 as obtained from (a) reduced analysis and (b) 4D Fourier transform. The color map is the same for both figures. 


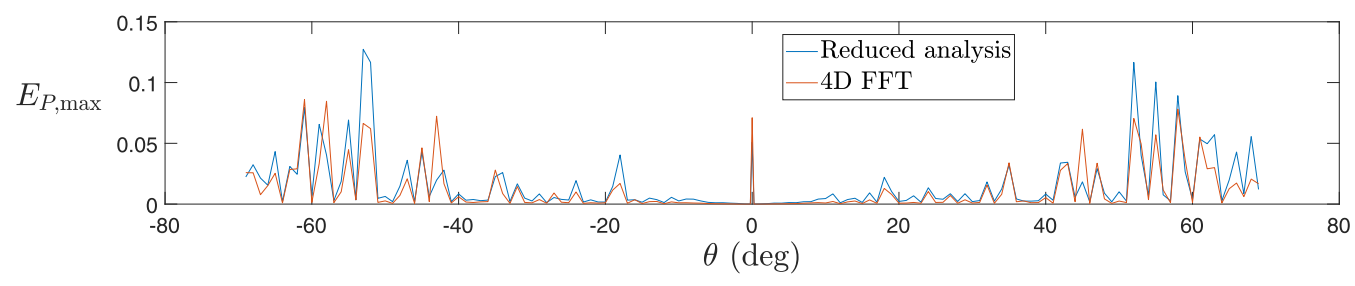

FIG. 18. Comparison of $E_{P, \max }(\theta)$ obtained from the reduced and full analyses for run R1.6.

we are taking the square of the sum of modes $\tilde{\mathbf{u}}(\mathbf{k}, \omega)$ within a given conical shell. Assuming we have $n$ such modes in a shell, the quantity $E_{K}(\theta, \omega)$ is

$$
\begin{aligned}
E_{K}(\theta, \omega) & =\frac{1}{2}\left(\tilde{\mathbf{u}}_{1}+\tilde{\mathbf{u}}_{2}+\cdots+\tilde{\mathbf{u}}_{n}\right) \cdot\left(\tilde{\mathbf{u}}_{1}+\tilde{\mathbf{u}}_{2}+\cdots+\tilde{\mathbf{u}}_{n}\right)^{*} \\
& =\frac{1}{2}\left(\left|\tilde{\mathbf{u}}_{1}\right|^{2}+\left|\tilde{\mathbf{u}}_{2}\right|^{2}+\cdots+\left|\tilde{\mathbf{u}}_{n}\right|^{2}\right)+\left(\operatorname{Re}\left\{\tilde{\mathbf{u}}_{1} \cdot \tilde{\mathbf{u}}_{2}^{*}\right\}+\operatorname{Re}\left\{\tilde{\mathbf{u}}_{1} \cdot \tilde{\mathbf{u}}_{3}^{*}\right\}+\cdots\right) .
\end{aligned}
$$

The first term in the final expression is the true kinetic energy in the shell and the second term is the sum of extra contributions. An equivalent development can be made involving the density perturbation modes $\tilde{\rho}^{\prime}(\mathbf{k}, \omega)$ contributing to the potential energy distribution $E_{P}(\theta, \omega)$ obtained from the reduced analysis. Fortunately, because the flow is turbulent, the phases of individual Fourier modes, whether they be corresponding to waves or to eddies, are "scrambled," which means that the overall sum of the cross terms is approximately zero. We can hence continue using this reduced analysis, keeping in mind that there is a small error and that the sum of $E_{K}(\theta, \omega)$ is $\sum_{\theta} \sum_{\omega} E_{K}(\theta, \omega) \approx E_{K}=(1 / 2)\left\langle|\mathbf{u}|^{2}\right\rangle$, where $E_{K}$ is the average kinetic energy in the numerical domain; i.e., these quantities are not exactly equal.

Similarly, the quantity $E_{K}(k, \theta, \omega)$ is formed by first summing $\hat{\mathbf{u}}(\mathbf{k})$ over circular annuli at constant equatorial angle $\theta$ and at constant $k$. The time Fourier transform of this sum is then computed and the subsequent squaring of $\mathcal{U}(k, \theta, \omega)$ introduces cross terms between the different Fourier modes that are present within the circular annulus. Hence, also $E_{K}(k, \theta, \omega)$ contains a small approximation. Moreover, the reduction of this quantity by summation over $k, E_{K}^{\prime}(\theta, \omega)=$ $\sum_{k} E_{K}(k, \theta, \omega)$, results in another approximation to the kinetic energy in $\theta-\omega$ space. Note that $E_{K}^{\prime}(\theta, \omega) \neq E_{K}(\theta, \omega)$, since $E_{K}(\theta, \omega)$ contains cross terms between all Fourier modes within a conical shell, which are significantly more than the sum of cross terms within the circular annuli making up the conical shell, contained in $E_{K}^{\prime}(\theta, \omega)$. For this reason, $E_{K}^{\prime}(\theta, \omega)$ is probably a more accurate measure than $E_{K}(\theta, \omega)$. In any case, the approximations should be small: For example, in global terms, for run R9.9, the error from the kinetic energy without shear modes $E_{K}^{\prime}$ is $1.2 \%$ when summing over $E_{K}(\theta, \omega)$ and $0.4 \%$ when summing over $E_{K}^{\prime}(\theta, \omega)$.

To assess the error in the reduced spatiotemporal analysis using a finer grain, we have computed the 4D Fourier transform of $\rho^{\prime}(\mathbf{x}, t)$ for the DNS run at lowest resolution, run R1.6. This has been done using a similar approach and postprocessing code compared to those used in Ref. [19]. This has led us to the true potential energy in frequency-wave-number space, $E_{P}(\mathbf{k}, \omega)$, which was then reduced to the $\theta-\omega$ space to obtain the exact version of $E_{P}(\theta, \omega)$. In Fig. 17, color maps of $E_{P}(\theta, \omega)$ for this run obtained from the reduced analysis [cf. Fig. 7(b)] and from this full 4D spatiotemporal analysis are given. Loosely speaking, it appears that the energy map obtained from the reduced analysis is a "noisy" version of the energy map obtained from the full analysis. The main features

TABLE II. Comparison of wave potential energy $E_{P, \omega \sim N}$ obtained from full and reduced analyses for run R1.6.

\begin{tabular}{lcccc}
\hline \hline$E_{P, \omega \sim N}$ (reduced) & $E_{P, \omega \sim N}($ full $)$ & $E_{P, \omega \sim N} / E_{K}^{\prime}($ reduced $)$ & $E_{P, \omega \sim N} / E_{K}^{\prime}$ (full) & Error \\
\hline 0.1334 & 0.1336 & 0.1454 & 0.1456 & $0.2 \%$ \\
\hline \hline
\end{tabular}



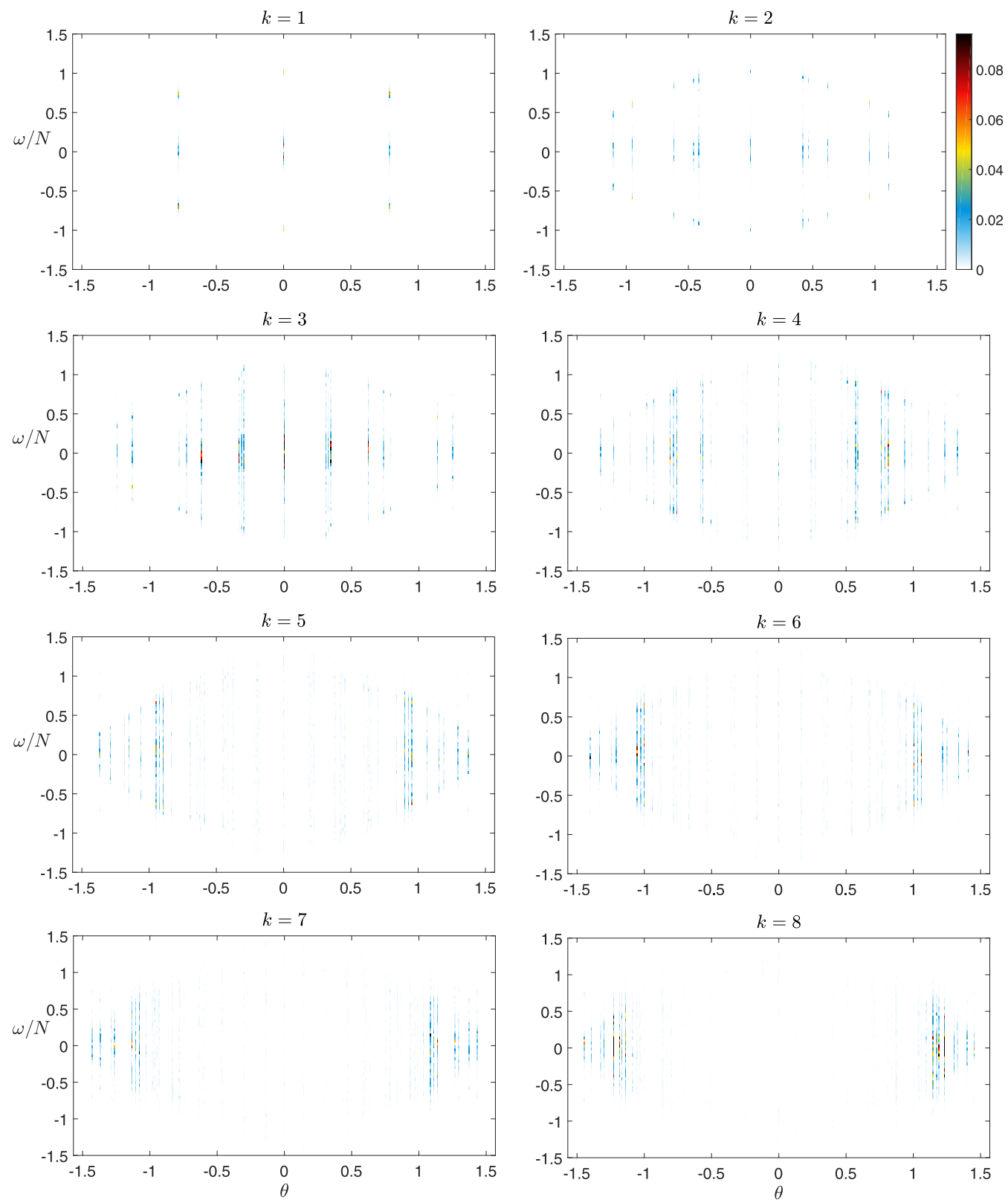

FIG. 19. Kinetic energy content $E_{K}(k, \theta, \omega)$ at the first eight wave numbers of run R9.9. The color map is the same across all color plots and is given on the plot corresponding to $k=2$; the upper limit of the color map has been chosen to make the wave energy visible in all plots, i.e., the energy in the vicinity of the dispersion relation. This results in the energy close to $\omega \approx 0$ (corresponding to vortex energy) saturating in a few cases as it is higher than this upper limit.

of the true $E_{P}(\theta, \omega)$ are recognizable in the reduced analysis version, for example, the dispersion relation and the additional two branches at higher frequency that appear for $\theta_{f \text {, min }}<|\theta|<\pi / 2$. Yet it is clear that significant deviations occur on a point-by-point basis. To further explore the effect of the approximation on the results presented herein, we recalculate the distribution of the maxima of 
$E_{P}(\theta, \omega)$ within the wave envelope as a function of $\theta$ (the wave envelope is defined using the same Doppler shift as before). The results are presented in Fig. 18. This figure highlights that the same trends are presented by the two versions of $E_{P, \max }(\theta)$, with most energy peaks of the true version being reproduced by the reduced analysis version at the same $\theta$ but with some variation of the height of the peaks.

Finally, we estimate the wave potential energy as obtained from the full analysis by summing $E_{P}(\theta, \omega)$ over the wave envelope. The results are shown in Table II where they are compared to the results of the reduced analysis. The comparison is very good and the error of the reduced analysis is very small for $E_{P, \omega \sim N}$. In conclusion, the full $4 \mathrm{D}$ analysis confirms that there are pointwise errors in the reduced analysis that result in added noise in $E_{P}(\theta, \omega)$. At the same time, the full analysis confirms that the main physical features observed using the reduced analysis, including the wave energy budget, are accurate, and therefore gives us confidence in the results presented previously.

\section{APPENDIX C: VARIATION IN WAVE ENERGY AT THE LARGEST WAVE NUMBERS}

In this Appendix, the distribution of wave and vortex kinetic energy over the largest scales, from $k=1$ to $k=8$, is considered. In Fig. 19, the kinetic energy maps $E_{K}(k, \theta, \omega)$ are presented, with $k=1$ up to $k=8$. It can be seen that, while the overall kinetic energy for every wave number is not constant, wave signatures (as well as vortex signatures) are present over all $k$. Moreover, the individual $\theta$ that are excited as a function of $k$ are now visible. Note that the number of wave vectors having a certain magnitude $k$ is limited due to the discrete numerical grid-in practice we have integer wave numbers - and so the number of $\theta$ is also limited. Clearly, due to this geometric constraint, the number of accessible $\theta$ is smallest for $k=1$ (only $\theta=0^{\circ}, 45^{\circ}, 90^{\circ}$ are accessible) and increases with increasing $k$. Considering individual $\theta$, i.e., individual waves, as a function of $k$, it seems that the energy content is roughly constant across all excited $\theta$ and across all $k$. However, the number of excited $\theta$ and so the number of peaks is not constant as a function of $k$ leading to a nonconstant overall wave energy from one map to the next, which may be due to the above geometric constraint. Overall, it seems reasonable to say that energy is distributed relatively evenly across all the waves excited in the range of wave numbers $k=\left[\begin{array}{ll}1 & 8\end{array}\right]$.

[1] F. Godeferd and C. Cambon, Detailed investigation of energy transfers in homogeneous stratified turbulence, Phys. Fluids 6, 2084 (1994).

[2] G. Spedding, F. Browand, and A. Fincham, Turbulence, similarity scaling, and vortex geometry in the wake of a towed sphere in a stably stratified fluid, J. Fluid Mech. 314, 53 (1996).

[3] M. Waite and P. Bartello, Stratified turbulence dominated by vortical motion, J. Fluid Mech. 517, 281 (2004).

[4] Y. Kimura and J. R. Herring, Energy spectra of stably stratified turbulence, J. Fluid Mech. 698, 19 (2012).

[5] J. Riley, R. Metcalfe, and M. Weissman, Direct numerical simulations of homogeneous turbulence in density-stratified fluids, in Nonlinear Properties of Internal Waves, edited by B. West, AIP Conf. Proc. No. 76 (AIP, New York, 1981), pp. 79-112.

[6] D. Lilly, Stratified turbulence and the mesoscale variability of the atmosphere, J. Atmos. Sci. 40, 749 (1983).

[7] J. Riley and M.-P. Lelong, Fluid motions in the presence of strong stable stratification, Annu. Rev. Fluid Mech. 32, 613 (2000).

[8] P. Bartello, Geostrophic adjustment and inverse cascades in rotating stratified turbulence, J. Atmos. Sci. 52, 4410 (1995).

[9] M. Waite and P. Bartello, The transition from geostrophic to stratified turbulence, J. Fluid Mech. 568, 89 (2006).

[10] C. Herbert, R. Marino, D. Rosenberg, and A. Pouquet, Waves and vortices in the inverse cascade regime of stratified turbulence with or without rotation, J. Fluid Mech. 806, 165 (2016). 
[11] P. Billant and J.-M. Chomaz, Self-similarity of strongly stratified inviscid flows, Phys. Fluids 13, 1645 (2001).

[12] E. Lindborg, The energy cascade in a strongly stratified fluid, J. Fluid Mech. 550, 207 (2006).

[13] G. Brethouwer, P. Billant, E. Lindborg, and J.-M. Chomaz, Scaling analysis and simulation of strongly stratified turbulent flows, J. Fluid Mech. 585, 343 (2007).

[14] E. Lindborg and G. Brethouwer, Stratified turbulence forced in rotational and divergent modes, J. Fluid Mech. 586, 83 (2007).

[15] J. Riley and E. Lindborg, Stratified turbulence: A possible interpretation of some geophysical turbulence measurements, J. Atmos. Sci. 65, 2416 (2008).

[16] A. Maffioli, Vertical spectra of stratified turbulence at large horizontal scales, Phys. Rev. Fluids 2, 104802 (2017).

[17] Q. Aubourg and N. Mordant, Nonlocal Resonances in Weak Turbulence of Gravity-Capillary Waves, Phys. Rev. Lett. 114, 144501 (2015).

[18] E. Yarom and E. Sharon, Experimental observation of steady inertial wave turbulence in deep rotating flows, Nat. Phys. 10, 510 (2014).

[19] H. Lam, A. Delache, and F. S. Godeferd, Partitioning waves and eddies in stably stratified turbulence, Atmosphere 11, 420 (2020).

[20] A. Ranjan, Inertial waves in rotating turbulence, Ph.D. thesis, University of Cambridge, Cambridge, UK, 2015.

[21] T. Le Reun, B. Favier, A. J. Barker, and M. Le Bars, Inertial Wave Turbulence Driven by Elliptical Instability, Phys. Rev. Lett. 119, 034502 (2017).

[22] A. Ranjan, P. Davidson, U. Christensen, and J. Wicht, Internally driven inertial waves in geodynamo simulations, Geophys. J. Int. 213, 1281 (2018).

[23] T. Le Reun, B. Favier, and M. Le Bars, Parametric instability and wave turbulence driven by tidal excitation of internal waves, J. Fluid Mech. 840, 498 (2018).

[24] P. Clark di Leoni, P. J. Cobelli, P. D. Mininni, P. Dmitruk, and W. H. Matthaeus, Quantification of the strength of inertial waves in a rotating turbulent flow, Phys. Fluids 26, 035106 (2014).

[25] P. Dmitruk and W. H. Matthaeus, Waves and turbulence in magnetohydrodynamic direct numerical simulations, Phys. Plasmas 16, 062304 (2009).

[26] M. Wheeler and G. N. Kiladis, Convectively coupled equatorial waves: Analysis of clouds and temperature in the wavenumber-frequency domain, J. Atmos. Sci. 56, 374 (1999).

[27] K. Dohan and B. Sutherland, Internal waves generated from a turbulent mixed region, Phys. Fluids 15, 488 (2003).

[28] A. Maffioli, P. Davidson, S. Dalziel, and N. Swaminathan, The evolution of a stratified turbulent cloud, J. Fluid Mech. 739, 229 (2014).

[29] P. Bonneton, J.-M. Chomaz, and E. Hopfinger, Internal waves produced by the turbulent wake of a sphere moving horizontally in a stratified fluid, J. Fluid Mech. 254, 23 (1993).

[30] A. Abdilghanie and P. Diamessis, The internal gravity wave field emitted by a stably stratified turbulent wake, J. Fluid Mech. 720, 104 (2013).

[31] T. Watanabe, J. J. Riley, S. M. de Bruyn Kops, P. J. Diamessis, and Q. Zhou, Turbulent/non-turbulent interfaces in wakes in stably stratified fluids, J. Fluid Mech. 797, R1 (2016).

[32] L. Liechtenstein, F. S. Godeferd, and C. Cambon, Nonlinear formation of structures in rotating stratified turbulence, J. Turbul. 6, N24 (2005).

[33] A. Delache, C. Cambon, and F. Godeferd, Scale by scale anisotropy in freely decaying rotating turbulence, Phys. Fluids 26, 025104 (2014).

[34] R. S. Rogallo, Numerical experiments in homogeneous turbulence, Tech. Rep. No. 81835, NASA Ames Research Center, Moffett Field, CA, United States, 1981.

[35] S. Almalkie and S. M. de Bruyn Kops, Kinetic energy dynamics in forced, homogeneous, and axisymmetric stably stratified turbulence, J. Turbul. 13, N29 (2012).

[36] P. Augier, P. Billant, and J.-M. Chomaz, Stratified turbulence forced with columnar dipoles: Numerical study, J. Fluid Mech. 769, 403 (2015). 
[37] A. Maffioli, G. Brethouwer, and E. Lindborg, Mixing efficiency in stratified turbulence, J. Fluid Mech. 794, R3 (2016).

[38] M. Waite and P. Bartello, Stratified turbulence generated by internal gravity waves, J. Fluid Mech. 546, 313 (2006).

[39] C. Rorai, P. D. Mininni, and A. Pouquet, Stably stratified turbulence in the presence of large-scale forcing, Phys. Rev. E 92, 013003 (2015).

[40] R. Marino, P. D. Mininni, D. L. Rosenberg, and A. Pouquet, Large-scale anisotropy in stably stratified rotating flows, Phys. Rev. E 90, 023018 (2014).

[41] A. Maffioli, Asymmetry of vertical buoyancy gradient in stratified turbulence, J. Fluid Mech. 870, 266 (2019).

[42] E. Kunze, A unified model spectrum for anisotropic stratified and isotropic turbulence in the ocean and atmosphere, J. Phys. Oceanog. 49, 385 (2019).

[43] J. J. Riley and S. M. de Bruyn Kops, Dynamics of turbulence strongly influenced by buoyancy, Phys. Fluids 15, 2047 (2003).

[44] A. Maffioli and P. Davidson, Dynamics of stratified turbulence decaying from a high buoyancy Reynolds number, J. Fluid Mech. 786, 210 (2016).

[45] C. Staquet and J. J. Riley, On the velocity field associated with potential vorticity, Dyn. Atmos. Oceans 14, 93 (1989).

[46] L. Smith and F. Waleffe, Generation of slow large scales in forced rotating stratified turbulence, J. Fluid Mech. 451, 145 (2002).

[47] C. J. Howland, J. R. Taylor, and C. P. Caulfield, Mixing in forced stratified turbulence and its dependence on large-scale forcing, J. Fluid Mech. 898, A7 (2020).

[48] A. Campagne, B. Gallet, F. Moisy, and P.-P. Cortet, Disentangling inertial waves from eddy turbulence in a forced rotating-turbulence experiment, Phys. Rev. E 91, 043016 (2015).

[49] K. L. Rowe, P. J. Diamessis, and Q. Zhou, Internal gravity wave radiation from a stratified turbulent wake, J. Fluid Mech. 888, A25 (2020).

[50] F. Godeferd and C. Staquet, Statistical modelling and direct numerical simulations of decaying stably stratified turbulence. Part 2. Large-scale and small-scale anisotropy, J. Fluid Mech. 486, 115 (2003). 